\title{
Organizing Time Exchanges: Lessons from Matching Markets*
}

\author{
Tommy Andersson`, Ágnes Cseh
}

First version: October, 2014. This version: March 21, 2020.

\begin{abstract}
This paper considers time exchanges via a common platform (e.g., markets for exchanging time units, positions at education institutions, and tuition waivers). There are several problems associated with such markets, e.g., imbalanced outcomes, coordination problems, and inefficiencies. We model time exchanges as matching markets and construct a non-manipulable mechanism that selects an individually rational and balanced allocation which maximizes exchanges among the participating agents (and those allocations are efficient). This mechanism works on a preference domain whereby agents classify the goods provided by other participating agents as either unacceptable or acceptable, and for goods classified as acceptable agents have specific upper quotas representing their maximum needs.
\end{abstract}

Keywords: market design; time balance; priority mechanism; non-manipulability.

JEL Classification: D82; D47.

\section{Introduction}

This paper considers time exchanges, i.e., markets where agents have initial endowments (that they wish to exchange with other agents), and there is a central organization overseeing the exchanges.

${ }^{*}$ We are grateful to four anonymous referees, Michael Ostrovsky (Co-Editor), Péter Biró, Jens Gudmundsson, and Flip Klijn for many useful and constructive comments. All authors gratefully acknowledge financial support from the Jan Wallander and Tom Hedelius Foundation (P2018-0100). Andersson is also grateful to Ragnar Söderbergs Stiftelse (E8/13) for financial support. Cseh was supported by the Hungarian Academy of Sciences (KEP-6/2019), its Momentum Programme (LP2016-3/2016), its János Bolyai Research Fellowship and OTKA grant K128611. Ehlers is grateful to the SSHRC (Canada) and the FRQSC (Québec) for financial support.

${ }^{\dagger}$ Lund University, Department of Economics. E-mail: tommy . andersson@nek.lu .se.

${ }^{\ddagger}$ Centre for Economic and Regional Studies, Institute of Economics, and University of Potsdam, Hasso Plattner Institute. E-mail: cseh.agnes@krtk.mta.hu.

${ }^{\S}$ Université de Montréal, Département de Sciences Économiques. E-mail: lars.ehlers@umontreal.ca.

ฯUniversity of Essex, Department of Economics. E-mail: albin.erlanson@essex .ac .uk. 
Three well-known time exchange markets are (i) the Erasmus program where more than 5,000 European higher education institutions across 37 countries participate to exchange students for shorter time periods; ${ }^{1}$ (ii) the US-based program The Tuition Exchange, Inc. (TTEI) where tuition waivers can be used by family members of faculty to attend colleges without paying tuition fees (Dur and Ünver, 2019); and (iii) time banks where groups of individuals and/or organizations set up common platforms to trade services among themselves (Cahn, 2011). Even if many exchange systems have been operating for decades, we argue that the absence of a centralized market structure is a source of inefficiency and that it may lead to "unbalanced" exchanges. These problems will ultimately threaten the long run sustainability of the exchange market. Given this observation, we propose a centralized market structure where exchanges are identified using a "priority mechanism" that satisfies a number of desirable properties on a reduced, yet relevant, preference domain.

As claimed above, there are reasons to believe that these exchange markets often are less efficient than centralized markets. First, centralized and computer-based clearing houses typically have lower overhead costs than their counterparts above. Second, it is challenging to identify and coordinate longer exchange cycles on "semi-centralized markets." By centrally organizing exchanges, a formal matching mechanism can identify and coordinate longer trading cycles based on reported preferences, which will lead to more efficient outcomes. Related to this, it is by no means clear that all reported information is taken into account when identifying exchanges in those markets, and this may, consequently, result in undesirable outcomes for the participating agents.

Inefficiencies aside, a well-documented obstacle for the long-term sustainability of time exchange markets is the fact that the exchanges are not necessarily "balanced." For example, in the Erasmus and the TTEI programs, the participating education institutions care about the balance of the incoming and outgoing students as there are no monetary transfers between participating institutions. Yet, there is still a documented imbalance in both these programs as described in Dur and Ünver (2019). Similarly, members of time banks are concerned about "time balance" since they do not wish to provide more time to the bank than they get back in return. Still, Ozanne (2010) concludes that time bank members often experience that time credits are comparatively easy to earn but harder to spend, i.e., that most members run a "time deficit." The lack of balance may discourage participation in these types of exchange markets, which ultimately threatens their long-term sustainability.

To overcome some of these problems associated with time exchange markets, this paper proposes

\footnotetext{
${ }^{1}$ See www .erasmusprogramme.compostwhat-is-the-erasmus-programme (retrieved September 1, 2019).
} 
a redesign whereby a central planner identifies exchanges based on a "priority mechanism" (discussed below). This mechanism identifies an allocation, i.e., a formal description of all exchanges, based on the information reported by the agents. The identified allocation only contains desirable (individually rational) exchanges; it is balanced and guarantees a maximal number of exchanges (the latter property implies efficiency on the considered preference domains). Given the interest in individually rational, efficient, and balanced allocations, a first observation is that such allocations always exist on the general preference domain. This follows since the allocation in which there are no exchanges (i.e., when all agents keep their initial endowments) is individually rational and satisfies balancedness. The conclusion then follows directly from the observation that the number of individually rational and balanced allocations is finite, and, consequently, there exists an allocation among those which is efficient.

Even if an allocation satisfying the specific properties of interest can be identified, two new problems arise. First, it is often natural to require that the matching mechanisms should be designed in such a fashion that it is in the best interest for all agents to report their preferences truthfully (nonmanipulability). This property is incompatible with individual rationality, efficiency, and balancedness on a general preference domain (Sönmez, 1999, Corollary 1). ${ }^{2}$ Second, because participating agents can be involved in multiple exchanges (e.g., education institutions may exchange multiple students, time bank members may receive services from several distinct members, etc.), it is not clear that it is easy for agents to generally rank any two distinct allocations. In timebanking, for example, is an allocation whereby the agent receives two hours of hairdressing, two hours of gardening and one hour of babysitting strictly better, equally good, or less preferred to an allocation where the agent receives one hour of hairdressing, one hour of gardening and three hours of housekeeping? Hence, it may be difficult for agents to report their preferences when they are allowed to be involved in multiple cyclical exchanges with a number of distinct agents.

We show that, if agent preferences satisfy certain conditions, the above two problems are no longer present. In some settings, the considered preference domain is clearly unrealistic (e.g., in exchange problems related to school choice). In other settings, it provides a reasonably good approximation. Given a few assumptions related to, e.g., monotonicity, the restricted domain only requires that each agent (a) partitions the other agents into two disjoint subsets containing agents that provide acceptable and unacceptable goods, and (b) specify an upper "exchange bound" for each acceptable agent. For

\footnotetext{
${ }^{2}$ This impossibility should come as no surprise given the results in, e.g., Hurwicz (1972), Green and Laffont (1979), Roth (1982), Alcalde and Barberà (1994), Barberà and Jackson (1995), or Schummer (1999).
} 
example, an education institution in the Erasmus program may classify the other participating institutions as either acceptable or unacceptable to send their students to (an agent's "horizontal" preference), but also an upper limit on how many students the institution at most wishes to send to each acceptable institution (an agent's "vertical" preference). The reduced preference domain facilitates agents' ability to report their preferences since they need only report the information in (a) and (b). Under all circumstances, it is most likely much easier to report preferences on this domain than on the general domain where agents need to report a complete ranking over all possible allocations. The considered reduced domain is an extension of the dichotomous domain that was popularized by Bogomolnaia and Moulin (2004). ${ }^{3}$

To solve the considered exchange problem, we define a "priority mechanism" and demonstrate that it can be formulated as a max-weight matching problem (Proposition 1). The definition of the priority mechanism is flexible since it can be adopted on both the restricted and the general preference domain. The main result shows that the priority mechanism is non-manipulable on the restricted preference domain and that it always makes a selection from the set of individually rational, maximal, and balanced allocations (Theorem 1). To prove this result, a number of novel graph theoretical techniques are needed. In particular, Appendix B demonstrates an equivalence result between the max-weight matching problem and a circulation-based maximization problem. ${ }^{4}$

\subsection{Related Literature}

Before providing some remarks on the existing market design literature, we note that this is not the first paper to use graph theoretical tools and, in particular, min-cost/max-weight formulations to solve matching problems. In the house allocation problem with dichotomous preferences, for example, Aziz (2018) formalizes a bipartite graph and solves for a max-weight matching. His graph construction is based on having houses on one side and agents on the other side of the market. This is in contrast to the approach in this paper, where agents are cloned into copies of themselves. Furthermore, our graph construction and the solution is more intricate since agents can be involved in multiple exchanges. Because finding a maximal allocation is more involved in our problem and a potential manipulation is more complex, graph theoretical tools are used to prove the non-manipulability result. This is not

\footnotetext{
${ }^{3}$ See Remark 2 for a discussion. Note also that the dichotomous domain is much smaller than the strict preference domain, but it is not a subset of the strict domain since indifference relations are allowed in the former but not in the latter domain.

${ }^{4}$ The max-weight matching problem is considered in the main part of the paper since it is more intuitive and, moreover, can be introduced using minimal notation.
} 
needed in Aziz (2018) because he considers a less complex optimization problem.

The considered exchange market shares many features with some classical markets, previously considered in the matching literature, including, e.g., housing markets (Scarf and Shapley, 1974; Abdulkadiroğlu and Sönmez, 1999; Aziz, 2018), organ markets (Roth et al., 2004; Biró et al., 2009; Ergin et al., 2017), one-to-one matching problems (Gale and Shapley, 1962), and markets for school seats (Abdulkadiroğlu and Sönmez, 2003; Kesten and Ünver, 2015). There are, however, substantial differences between these problems and the one considered in this paper. For example, our model allows agents to be involved in multiple exchanges. In the school choice problem and the kidney exchange problem, in contrast, students are allocated at most one school seat, and each patient-donor pair is involved in at most one kidney exchange, respectively. Furthermore, in many matching problems including, e.g., the school choice problem and the house allocation problem, agents' (reported) preferences are typically strict, and indifference relations are consequently not allowed (while the kidney exchange problem is often defined on a dichotomous domain). Generalizations to allow for a weak preference structure have recently been proposed by Alcalde-Unzu and Molis (2011) and Jaramillo and Manjunath (2012). However, both these papers only allow agents to trade at most one object.

By organizing the considered type of time exchanges as a matching market, it will have the structure of a many-to-many matching market. Such markets have previously been considered by, e.g., Echenique and Oviedo (2006), Konishi and Ünver (2006), and Hatfield and Kominers (2016). The papers closest to the model investigated in this study are Athanassoglou and Sethuraman (2011), Aziz (2016), Biró et al. (2017a,b) and Manjunath and Westkamp (2019), which we describe next.

Athanassoglou and Sethuraman (2011) and Aziz (2016) consider a housing market where initial endowments as well as allocations are described by a vector of fractions of the houses in the economy. The fractional setting makes it possible to analyze, e.g., efficiency based on (first-order) stochastic dominance, and it is demonstrated that the efficiency and fairness notions of interest conflict with non-manipulability. Even if a similar impossibility is present in our model, the fractional setting is analyzed using different axioms and mechanisms. In addition, Athanassoglou and Sethuraman (2011) and Aziz (2016) are unable to find any positive results related to non-manipulability in their considered reduced preference domains.

Biró et al. (2017a) consider, as does this paper, a model where agents are endowed with multiple units of an indivisible and agent-specific good, searching for balanced allocations. In their reduced preference domain, agents have responsive preferences over consumption bundles. In this reduced 
domain, they demonstrate that, for general capacity configurations, no mechanism satisfies individual rationality, efficiency, and non-manipulability. Given this negative finding, they characterize the capacity configurations for which individual rationality, efficiency and non-manipulability are compatible. They also demonstrate that, for these capacity configurations, their mechanism is the unique mechanism that satisfies all three properties of interest. Hence, the main difference between this paper and Biró et al. (2017a) is that they consider a different preference domain and, consequently, need a different mechanism to escape the impossibility result.

Manjunath and Westkamp (2019) have independently considered a model of shift exchange that is closely related to the one considered here. In terms of our application to time banks, the model of Manjunath and Westkamp allows each agent to supply distinct services as opposed to our model where each agent supplies the same service in multiple units. Like us, Manjunath and Westkamp require exchange to be balanced and consider a responsive preference domain classifying individual services as either unacceptable and acceptable. Since agents are allowed to be indifferent between different services, their model is able to handle multiple copies of the same service. Manjunath and Westkamp (2019) show that a priority mechanism over the set of all individually rational allocations is efficient and strategy-proof. The main differences between our work and that of Manjunath and Westkamp are that (a) their model is more general in that it allows agents to have distinct services whereas each agent in our model has a specific service that comes in multiple copies, and (b) their priority mechanism is only guaranteed to be Pareto-efficient while our mechanism satisfies the more demanding efficiency criterion of maximality (and as we show in Example 3 allocations from the two mechanisms can indeed be quite different). Finally, (c) for the non-manipulability result our proof techniques are very different and are complementary.

\subsection{Outline of the Paper}

The remaining part of the paper is outlined as follows. Because exchange problems related to tuition exchange and the Erasmus program are well-documented in the matching literature (see, e.g., Biró, 2017; Delacrétaz, 2019; Dur and Kesten, 2018; Dur and Ünver, 2019), Section 2 will introduce a problem that has received much less attention, namely the timebanking problem. This section gives a detailed introduction to timebanking and provides some descriptive statistics of the time banks associated with TimeBanks USA. Section 3 introduces the general theoretical exchange framework and some basic definitions. Section 4 introduces priority mechanisms. The main results are presented 
in Section 5. Section 6 discusses the main findings of the paper. Finally, Section 7 concludes the paper. All proofs are relegated to the Appendix.

\section{Timebanking}

Even if concepts closely related to timebanking dates back to the 19th century, timebanking was popularized and pioneered in the 1990s by Edgar Cahn and Martin Simon in the United States and the United Kingdom, respectively. However, it took another 20 years before the concept of timebanking had a serious impact in society. Dash and Sandhu (2018) report that the first time bank in the United Kingdom was set up in 1996, but only 2,200 persons had joined a time bank by 2003. Eight years later, after additional experimentation, learning, and expansion, there were around 30,000 registered members in the United Kingdom, 30,000 registered members in the United States, and an additional 100,000 members scattered across 34 countries (Cahn, 2011). This number has continued to grow. In 2014, there were around 35,000 members in the United Kingdom and even more in the United States. ${ }^{5}$

As explained in the Introduction, a time bank is a group of individuals and/or organizations in a local community that set up a common platform to trade services among themselves. For example, a gardener who supplies two hours of time may, for example, receive two hours of child care in return for his gardening services. Ozanne (2010) reported that the most commonly exchanged services included gardening, giving lifts, befriending, do-it-yourself jobs, dog walking, and computer training. Members of a time bank earn one time credit for each time unit they supply to members of the bank. The earned credit can be spent to receive services from other members of the bank. ${ }^{6}$

In most time banks, a broker is employed to manage the bank, maintain the database, record transactions, recruit new members, etc. (Seyfang, 2003, 2004; Williams, 2004). A “matching system” helps the broker to coordinate requests for services with those who can provide them. In some time banks, this "system" is simple and the broker manually matches requests with offers (Seyfang, 2003). A few large time banks, including TimeBanks USA and Timebanking UK, have developed their own computer software where members can see what other members offer and keep track of their own activity. For example, the software used by TimeBanks USA (called Community Weaver 3) allows members to register their talents in 11 different categories including, e.g., education, transportation, business services, recreation, and companionship. Each of these categories also has subcategories. The category

\footnotetext{
${ }^{5}$ These figures are from Boyle and Bird (2014) or www .timebanks . org (retrieved 2019-02-05).

${ }^{6}$ Very few time banks are not based on a "one-for-one" time system, meaning that members of the time bank need not get one unit of time back for each unit of time they supply (Croall, 1997).
} 
"education," for example, contains subcategories such as advocacy, computers, languages, finances, and tutoring. When a member has registered her talents, she can start offering her services and begin making requests. An offer is a formal registration on the online platform that enables other members to see and request her talents. If a member approves a request, she receives the agreed amount of time credits and the member who receives the service is credited by the same amount of time credits.

Even if some time banks take advantage of computer software, it is difficult to coordinate longer trading cycles as members only can see their own activity. As a consequence, time bank members are naturally restricted to bilateral exchanges. Furthermore, it may be an obstacle for time bank members to report too "detailed" preferences if they potentially can be involved in multiple exchange cycles. This is also one of the reasons for considering a reduced preference domain (informally described in the Introduction and formally defined in Section 3.2) whereby each time bank member partitions the other members of the bank (or, equivalently, the services they provide) into two disjoint subsets containing acceptable and unacceptable members, and specify an upper time bound for each acceptable member. The former condition reflects that an agent is not necessarily interested in all services provided in the bank. The latter condition captures the idea that an agent may, for example, be interested in at most one haircut but can accept up to 10 hours of babysitting. This preference domain clearly facilitates time bank members' ability to report their preferences, and it captures certain aspects of existing time banks, e.g., that members classify their services in various categories and that members request certain categories of services.

\subsection{Timebanking and Society}

The types of services provided by members of time banks, as exemplified above, are typically available and priced on competitive markets. Given this observation, it is natural to ask: what role do time banks play in society and what explains the existence of time banks in a society where monetary transfers are available?

One potential explanation may be the documented fact (Collom, 2007; Seyfang, 2003; Seyfang and Smith, 2002) that time banks are very successful in attracting participants from socially excluded groups and low-income communities, e.g., people on benefit programs, from low-income households, etc. $^{7}$ That is, because most time bank members have small social networks and, in many cases, also

\footnotetext{
${ }^{7}$ Most studies in the existing literature, consequently, focus on socially excluded and low-income groups. An exception is Ozanne (2010) where it is demonstrated that time banks have provided high benefits in the form of social, human, physical and cultural capital also within affluent groups.
} 
lack both income and employment, timebanking is one way to be included in a social network and to increase welfare. Another way of expressing this observation is that persons from socially excluded groups often have a scarcity of traditional means of payment (i.e., "money"), but can gain access to an alternative currency by joining a time bank (i.e., "time credits"). This will increase their purchasing power. In fact, Collom (2007) has documented that the single most important reason for joining a time bank is to expand purchasing power through an alternative currency.

Another potential explanation is deeply rooted in the philosophy of timebanking. As stated by one of its proponents Cahn (2000), different fairness and equality notions are embedded in the five core values of timebanking: assets, redefining work, reciprocity, social networks, and respect. For example, and as explained above, one of the fundamental ideas in timebanking is that one hour of service generates one time credit regardless of the provider or the nature of the service performed. Consequently, even if services provided by time bank members are valued and priced differently on competitive markets, the timebanking philosophy prescribes that human beings share fundamental equality and their services should therefore be "priced" equally (through a one-for-one time exchange system).

Furthermore, an integral part of these core values is to extend social networks, increase informal neighborhood support, and develop reciprocal relationships (Cahn, 2000). One way to reach these goals is to provide a marketplace that is "less anonymous" than competitive markets and to more explicitly set up a market where people can meet physically and exchange services. In fact, Seyfang (2003) found that persons join time banks to meet other members, to help other members, to get more involved in the local neighborhood, etc. Similar motives are recorded by, e.g., Collom (2007) and Caldwell (2000). Related to this, Boyle et al. (2006) showed that time banks not only help their members to extend their social networks but also that time banks are an effective way of developing reciprocal relationships between members in the bank.

\subsection{Organization and Descriptive Statistics of TimeBanks USA}

To the best of our knowledge, there exists no public database that provides detailed statistics about time banks worldwide. To give the reader at least some idea about, e.g., the size and number of transactions in a time bank, this section provides some descriptive statistics of TimeBanks USA, the largest time bank operating in the United States. ${ }^{8}$ The data are from the period April 1, 2015 (when

\footnotetext{
${ }^{8}$ All data and documentation related to TimeBanks USA stated in this section are available in the Online Appendix.
} 
Community Weaver 3 was launched) to January 15, 2019 (when we collected the data).

TimeBanks USA currently has 107 branches in the United States spread out over 33 states, and it also operates in Australia, Canada, France, Greece, Guatemala, Israel, New Zealand, South Africa, South Korea, and the United Kingdom (see Table 1). Even if not all registered branches are active, Table 2 provides some more detailed information about the active branches. As can be seen from the table, a time bank located in the United States has on average around 100 members and has, on average, performed 1,958 trades since April 2015. These trades involved on average 7,736 time units per branch, meaning that each time exchange was, on average, for 3.95 hours. As also can be seen from Table 2, the average time bank in the United States had 115.1 registered active offers and 115.5 active requests on January 15, 2019. The figures from Table 2, therefore, roughly translates to each member, in an active branch, having, on average, had one active offer and one active request on January 15, 2019.

Table 1: Descriptive data of TimeBanks USA.

\begin{tabular}{lrrr}
\hline Country & Number of branches & Active branches & Represented in states/provinces/regions \\
\hline USA & 107 & 84 & 33 out of 50 \\
New Zealand & 30 & 28 & 7 out of 16 \\
Canada & 11 & 9 & 5 out of 10 \\
Other countries & 8 & 7 & - \\
\hline
\end{tabular}

* The data were collected from www.timebanks . org on 2019-01-15 and are available in the Online Appendix.

Table 2: Mean summary statistics for the active time banks in Table 1.

\begin{tabular}{lrrrrr}
\hline Country & Number of members & Number of exchanges & Number of hours exchanged & Active offers & Active requests \\
\hline USA & $98.9(105.9)$ & $1,957.8(5,034.9)$ & $7,736.0(25,509.6)$ & $115.1(748.9)$ & $115.5(746.6)$ \\
New Zealand & $158.7(189.7)$ & $1,913.4(2,798.6)$ & $10,568.9(29,138.0)$ & $25.9(23.7)$ & $28.2(28.1)$ \\
Canada & $64.1(64.9)$ & $187.1(255.3)$ & $600.9(1,019.7)$ & $34.4(41.6)$ & $27.9(38.9)$ \\
Other countries & $113.7(233.5)$ & $1,464.3(3,623.4)$ & $5,401.7(13,694.3)$ & $1.6(2.1)$ & $2.0(3.0)$ \\
\hline
\end{tabular}

* All values are mean values (standard deviation within brackets).

\section{The Model and Basic Definitions}

This section introduces the exchange problem together with some definitions and axioms.

\subsection{Agents, Bundles, and Allocations}

Let $N=\{1, \ldots, n\}$ denote the finite set of agents. Each agent $i \in N$ is endowed with $t_{i} \in \mathbb{N}$ units of a good (or service) which can be used to exchange goods (or services) with agents in $N$. One 
can, for example, think of these goods as positions at education institutions, tuition waivers, or time units. Let $t=\left(t_{1}, \ldots, t_{n}\right)$ denote the vector of endowments. Because the exact nature of the goods is of secondary interest, the problem will be described in terms of the number of goods that an agent receives from and provides to other agents in $N$. Let $x_{i j}$ denote the number of goods that agent $i \in N$ receives from agent $j \in N$, or, equivalently, the number of goods that agent $j$ provides to agent $i$. Here, $x_{i i}$ represents the number of goods that agent $i \in N$ receives from herself. It is assumed that $x_{i j}$ belongs to the set $\mathbb{N}_{0}$ of non-negative integers that includes $0 .{ }^{9}$

The goods that agent $i \in N$ receives from the agents in $N$ can be described by the bundle $x_{i}=$ $\left(x_{i 1}, \ldots, x_{i n}\right)$. The bundle where agent $i \in N$ keeps his initial endowment is denoted by $\omega_{i}$ (where $\omega_{i i}=t_{i}$ and $\omega_{i j}=0$ for $\left.j \neq i\right)$. An allocation $x=\left(x_{1}, \ldots, x_{n}\right)$ is a collection of $n$ bundles (one for each agent in $N$ ).

An allocation is feasible if:

$$
\begin{aligned}
& \sum_{j=1}^{n} x_{i j}=t_{i} \text { for all } i \in N, \\
& \sum_{j=1}^{n} x_{j i}=t_{i} \text { for all } i \in N .
\end{aligned}
$$

This means any agent $i$ receives the same number of goods from other agents that the agent supplies to other agents (recall that an agent can keep parts of his initial endowment). In this sense, any feasible allocation satisfies the balancedness conditions (1) and (2). In the remaining part of the paper, it is understood that any allocation is feasible. Let $\mathcal{F}$ denote the set of all feasible allocations.

\subsection{Preferences and Preference Domains}

A preference relation for agent $i \in N$ is a complete and transitive binary relation $R_{i}$ over feasible bundles such that $x_{i} R_{i} x_{i}^{\prime}$ whenever agent $i$ finds bundle $x_{i}$ at least as good as bundle $x_{i}^{\prime}$. Let $P_{i}$ and $I_{i}$ denote the strict and the indifference part of $R_{i}$, respectively. Let $\mathcal{R}_{i}$ denote the set of all preference relations of agent $i \in N$. A (preference) profile $R$ is a list of individual preferences $R=\left(R_{1}, \ldots, R_{n}\right)$. The general domain of profiles is denoted by $\mathcal{R}=\mathcal{R}_{1} \times \cdots \times \mathcal{R}_{n}$. A profile $R \in \mathcal{R}$ may also be written as $\left(R_{i}, R_{-i}\right)$ when the preference relation $R_{i}$ of agent $i \in N$ is of particular importance.

\footnotetext{
${ }^{9}$ In the timebanking application, one can think of $x_{i j}$ as a representation of standardized time units, e.g., 0 minutes for zero units, 30 minutes for one unit, 60 minutes for two units, etc.
} 
A restricted preference domain $\tilde{\mathcal{R}}=\tilde{\mathcal{R}}_{1} \times \cdots \times \tilde{\mathcal{R}}_{n} \subset \mathcal{R}$ will be considered for our main results. As explained in the Introduction, this restricted domain is based on the idea that any preference relation $R_{i} \in \tilde{\mathcal{R}}_{i}$ :

(a) partitions the set of agents $N \backslash\{i\}$ into two disjoint sets containing acceptable and unacceptable agents (i.e., agents that provide acceptable and unacceptable goods), denoted by $A_{i}\left(R_{i}\right) \subseteq$ $N \backslash\{i\}$ and $U_{i}\left(R_{i}\right)=N \backslash\left(A_{i}\left(R_{i}\right) \cup\{i\}\right)$, respectively, and;

(b) associates with each acceptable agent $j \in A_{i}\left(R_{i}\right)$ an upper bound $\bar{t}_{i j} \in \mathbb{N}_{0}$ on the number of goods that agent $i$ at most would like to receive from agent $j$.

Here, one may interpret (a) as agent $i$ 's "horizontal preference" over acceptable and unacceptable agents, and (b) as agent $i$ 's "vertical preference" representing the maximal demand of each good provided by an acceptable agent. Then, for agent $i \in N$, the preference relation $R_{i}$ belongs to $\tilde{\mathcal{R}}_{i}$ if for any allocations $x$ and $y$ :

(i) $\omega_{i} P_{i} x_{i}$ if $x_{i k}>0$ for some $k \in U_{i}\left(R_{i}\right)$ or $x_{i j}>\bar{t}_{i j}$ for some $j \in A_{i}\left(R_{i}\right)$,

(ii) $x_{i} I_{i} y_{i}$ if both $\omega_{i} P_{i} x_{i}$ and $\omega_{i} P_{i} y_{i}$,

(iii) $y_{i} P_{i} x_{i}$ if $y_{i} R_{i} \omega_{i}, x_{i} R_{i} \omega_{i}$ and $\sum_{j \in A_{i}\left(R_{i}\right)} y_{i j}>\sum_{j \in A_{i}\left(R_{i}\right)} x_{i j}$, or;

(iv) $y_{i} I_{i} x_{i}$ if $y_{i} R_{i} \omega_{i}, x_{i} R_{i} \omega_{i}$ and $\sum_{j \in A_{i}\left(R_{i}\right)} y_{i j}=\sum_{j \in A_{i}\left(R_{i}\right)} x_{i j}$

The first condition states that an agent strictly prefers not to be involved in an exchange if (a) the agent receives goods from unacceptable agents or (b) the number of goods provided by an acceptable agent exceeds the upper bound. The second condition means that an agent is indifferent between any two bundles where (a) and/or (b) holds. The last two conditions reflect a monotonicity property and state that an agent weakly prefers bundles with weakly more acceptable agents whenever bundles do not contain any unacceptable agents and as long as the bounds $\bar{t}_{i j}$ are not exceeded for the acceptable agents contained in the bundle.

Remark 1. For the restricted domain $\tilde{\mathcal{R}}$, a report $R_{i}$ for agent $i \in N$ is given by a set of acceptable agents $A_{i}\left(R_{i}\right)$ together with an upper bound $\bar{t}_{i j}$ for each $j \in A_{i}\left(R_{i}\right)$. An equivalent formulation of the reported preference for agent $i \in N$ is a vector $\bar{t}_{i}=\left(\bar{t}_{i 1}, \ldots, \bar{t}_{i n}\right) \in \mathbb{N}_{0}^{n}$ where $\bar{t}_{i i}=t_{i}$. Then $\bar{t}_{i j}=0$ stands for $j \in U_{i}\left(R_{i}\right)$, i.e., agent $i$ is willing to accept at most zero units from agent $j$. Whether the first or the second formulation is used is just a matter of choice. 
Remark 2. For any agent $i \in N$ and $R_{i} \in \tilde{\mathcal{R}}_{i}$, the preference $R_{i}$ is dichotomous over single agents because agents are partitioned into agents that provide acceptable and unacceptable goods. The preference $R_{i}$ is polychotomous over bundles in the following way: for any $h=0,1, \ldots, \min \left\{t_{i}, \sum_{j \in A_{i}\left(R_{i}\right)} \bar{t}_{i j}\right\}=$ $m$, all allocations $x$ and $y$ such that $x_{i j} \leq \bar{t}_{i j}$ and $y_{i j} \leq \bar{t}_{i j}$ for all $j \in A_{i}\left(R_{i}\right), x_{i k}=0=y_{i k}$ for all $k \in U_{i}\left(R_{i}\right)$ and $\sum_{j \in A_{i}\left(R_{i}\right)} y_{i j}=h=\sum_{j \in A_{i}\left(R_{i}\right)} x_{i j}$ are ranked indifferent by $R_{i}$. Let $\mathcal{I}(h)$ denote this indifference class. Then under $R_{i}$ all allocations in $\mathcal{I}(m)$ are strictly preferred to all allocations in $\mathcal{I}(m-1)$, and in general, for $h=1, \ldots, m$, under $R_{i}$ all allocations in $\mathcal{I}(h)$ are strictly preferred to all allocations in $\mathcal{I}(h-1)$. Thus, $R_{i}$ contains $m+2$ indifference classes (where $\mathcal{I}(0)=\left\{\omega_{i}\right\}$ and $\omega_{i}$ is strictly preferred to all allocations where the agent receives goods from unacceptable agents or the number of goods provided by an acceptable agent exceeds the upper bound). In this sense, preferences belonging to $\tilde{\mathcal{R}}_{i}$ are polychotomous over bundles (where the upper bounds are incorporated) and at the same time dichotomous over single agents.

\subsection{Axioms and Mechanisms}

Recall that $\mathcal{F}$ denotes the set of all feasible allocations. Allocation $x \in \mathcal{F}$ is individually rational if, for all $i \in N, x_{i} R_{i} \omega_{i}$. Allocation $x \in \mathcal{F}$ Pareto dominates allocation $x^{\prime} \in \mathcal{F}$ if $x_{i} R_{i} x_{i}^{\prime}$ for all $i \in N$ and $x_{j} P_{j} x_{j}^{\prime}$ for some $j \in N$. An allocation is efficient if it is not Pareto dominated by any feasible allocation. An allocation $x$ is maximal at $R$ if $\sum_{i \in N} \sum_{j \in A_{i}\left(R_{i}\right)} x_{i j} \geq \sum_{i \in N} \sum_{j \in A_{i}\left(R_{i}\right)} x_{i j}^{\prime}$ for all individually rational allocations $x^{\prime}$. All individually rational and maximal allocations at profile $R \in \tilde{\mathcal{R}}$ are gathered in the set $\mathcal{X}(R) \subset \mathcal{F}$. Note that $\mathcal{X}(R) \neq \emptyset$ for all $R \in \tilde{\mathcal{R}}$ and that any $x \in \mathcal{X}(R)$ is efficient. ${ }^{10}$

A mechanism $\varphi$ with domain $\tilde{\mathcal{R}}$ chooses for any profile $R \in \tilde{\mathcal{R}}$ a feasible allocation $\varphi(R) \in \mathcal{F}$. Mechanism $\varphi$ is manipulable at profile $R \in \tilde{\mathcal{R}}$ by an agent $i \in N$ if there exists $R_{i}^{\prime}$ such that $R^{\prime}=\left(R_{i}^{\prime}, R_{-i}\right) \in \tilde{\mathcal{R}}$, and for $x=\varphi(R)$ and $x^{\prime}=\varphi\left(R^{\prime}\right)$ we have $x_{i}^{\prime} P_{i} x_{i}$. If mechanism $\varphi$ is not manipulable by any agent $i \in N$ at any profile $R \in \tilde{\mathcal{R}}$, then $\varphi$ is non-manipulable (on the domain $\tilde{\mathcal{R}}$ ).

\section{Priority Mechanisms}

Any priority mechanism uses a priority-ordering. This ordering may be deduced from a lottery or from a schematic update based on previous allocation rounds. Let $\pi: N \mapsto N$ be an exogenously given

\footnotetext{
${ }^{10}$ If $x$ is not efficient, then there exists an individually rational allocation $x^{\prime}$ such that $x_{i}^{\prime} R_{i} x_{i}$ for all $i \in N$ and $x_{j}^{\prime} P_{j} x_{j}$ for some $j \in N$. But then $\sum_{i \in N} \sum_{j \in A_{i}\left(R_{i}\right)} x_{i j}<\sum_{i \in N} \sum_{j \in A_{i}\left(R_{i}\right)} x_{i j}^{\prime}$ meaning that $x$ is not maximal, which is a contradiction.
} 
priority-ordering where the highest-ranked agent is $i \in N$ with $\pi(i)=1$, the second-highest-ranked agent is $i^{\prime} \in N$ with $\pi\left(i^{\prime}\right)=2$, and so on.

Given $R \in \tilde{\mathcal{R}}, i \in N$ and $\mathcal{Z}^{*} \subseteq \mathcal{X}(R)$, allocation $x \in \mathcal{Z}^{*}$ belongs to the set $\mathcal{X}^{i, \mathcal{Z}^{*}}(R)$ if $x_{i} R_{i} x_{i}^{\prime}$ for all $x^{\prime} \in \mathcal{Z}^{*}$, i.e., if allocation $x$ is weakly preferred to any allocation in the set $\mathcal{Z}^{*}$ under preference $R_{i}$. In the special case where the set $\mathcal{Z}^{*}$ is based on the choice made by some agent $i^{\prime} \neq i$ for some profile $R \in \tilde{\mathcal{R}}$, i.e., where $\mathcal{Z}^{*}=\mathcal{X}^{i^{\prime}, \mathcal{Z}^{* *}}(R)$ for some $\mathcal{Z}^{* *} \subseteq \mathcal{X}(R)$, the set $\mathcal{X}^{i, \mathcal{Z}^{*}}(R)$ is denoted by $\mathcal{X}^{i, i^{\prime}}(R)$.

Definition 1. An allocation $x \in \mathcal{X}(R)$ is agent- $i$-optimal at profile $R \in \tilde{\mathcal{R}}$ if $x \in \mathcal{X}^{i, \mathcal{X}(R)}(R)$.

Note the difference between the sets $\mathcal{X}^{i, \mathcal{X}(R)}(R)$ and $\mathcal{X}^{i, \mathcal{Z}^{*}}(R)$. The former set contains all agent $i$ 's most preferred allocations in the set $\mathcal{X}(R)$ whereas the latter set contains all agent $i$ 's most preferred allocations in a subset $\mathcal{Z}^{*}$ of $\mathcal{X}(R)$.

Definition 2. Let $\pi$ be a priority-ordering and $N=\left\{i_{1}, \ldots, i_{n}\right\}$ be such that $\pi\left(i_{k}\right)=k$ for all $k=1, \ldots, n$. Then $x \in \mathcal{X}(R)$ is a $\pi$-priority allocation at profile $R \in \tilde{\mathcal{R}}$ if:

(i) $x$ belongs to $\mathcal{X}^{i_{1}, \mathcal{X}(R)}(R)$,

(ii) $x$ belongs to $\mathcal{X}^{i_{k}, i_{k-1}}(R)$ for all $k=2, \ldots, n$.

One way to think about the set of priority allocations is the following. First, the highest-ranked agent identifies all his most preferred allocations in the set $\mathcal{X}(R)$. Then, the agent with the second-highest priority identifies all his most preferred allocations in the set identified by the highest-ranked agent; then, the agent with the third-highest priority identifies all his most-preferred allocations in the set identified by the second highest ranked agent, and so on. Formally, this means that, if $x$ is a $\pi$-priority allocation, then:

$$
x \in \mathcal{X}^{i_{n}, i_{n-1}}(R) \subseteq \mathcal{X}^{i_{n-1}, i_{n-2}}(R) \subseteq \ldots \subseteq \mathcal{X}^{i_{2}, i_{1}}(R) \subseteq \mathcal{X}^{i_{1}, \mathcal{X}(R)}(R) \subseteq \mathcal{X}(R)
$$

Note that a priority allocation is agent- $i$-optimal for the agent $i \in N$ with $\pi(i)=1$. Moreover, all agents in $N$ are, by construction, indifferent between all allocations in the set $\mathcal{X}^{i_{n}, i_{n-1}}(R)$.

Definition 3. A mechanism $\varphi$ is a priority mechanism if there exists a priority-ordering $\pi$ such that for all profiles $R \in \tilde{\mathcal{R}}$ the mechanism $\varphi$ selects a $\pi$-priority allocation from the set $\mathcal{X}(R)$.

Since a priority mechanism always makes a selection from the set $\mathcal{X}(R)$, it chooses an individually rational, maximal, and balanced allocation (which is efficient). 


\section{Results}

As we show in Section 6, it is impossible to construct an individually rational, efficient, and nonmanipulable mechanism on the general domain $\mathcal{R}$. Our first main result demonstrates that this impossibility can be avoided on the restricted domain $\tilde{\mathcal{R}}$ if exchanges are based on a priority mechanism.

Theorem 1. Any priority mechanism with domain $\tilde{\mathcal{R}}$ is non-manipulable.

In most settings, proving non-manipulability of a priority mechanism is rather straightforward, see, e.g., Svensson (1994). In our setting with multiple objects and potentially different number of objects, the scope for manipulation is much larger. The maximal set of allocations changes in a complex manner if one agent reports something slightly different. A key observation in establishing nonmanipulability of the priority mechanism is that it is enough to ensure that no agent can ever gain by stating that she wants fewer units from an acceptable agent than she actually desires. This significantly reduces the possible scope of manipulations.

In order to show that no agent can ever gain by reducing the number of desired copies from an acceptable agent, we formulate a circulation flow network corresponding to the allocation in the priority mechanism (see Appendix B for details). This enables us to keep track of changes from potential manipulations using the circulation formulation. It is straightforward to see that the overall value in the associated optimization problem will decrease, since the feasible set shrinks. It is, however, a non-trivial argument to show that also the agent who reduces her capacity will not increase the flow through her nodes. For details of the argument and the construction of the circulation flow network, see Appendix B.

In Proposition 1 below, it is demonstrated that a priority mechanism can be formulated as a maxweight matching problem with vertex and edge capacities. Thereby we know that there exists an efficient way of computing the allocations chosen by the priority mechanism. The max-weight matching problem is a special case of the well-known network flow problem; thus, it is somewhat easier to describe than the circulation flow network formulation introduced in Appendix B. To formulate the max-weight matching problem, a bipartite graph needs to be defined, with edge capacities and vertex quotas. The edge weights will be introduced later.

Definition 4. For any profile $R \in \tilde{\mathcal{R}}$, the bipartite graph $g=(N, M, E, u, q)$ is defined by two disjoint sets of vertices, $N$ and $M$, a set of edges, $E$, a profile of capacities $u=(u(i, l))_{(i, l) \in E}$ on each edge, and a profile of quotas on the vertices $q=(q(i))_{i \in V}$. These are defined by: 


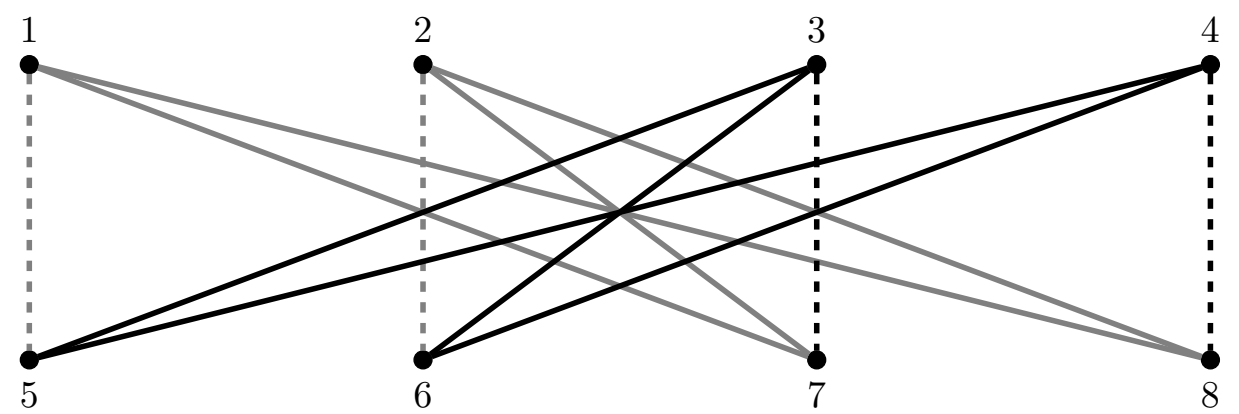

Figure 1: Edge capacity 1 is color-coded by gray, while capacity 2 is denoted by black edges. The edges connecting two copies of the same agent are marked by dashed lines. The four vertices on the left have quota 1 , while the four vertices on the right have quota 2.

(i) $N=\{1, \ldots, n\}$,

(ii) $M=\{n+1, n+2, \ldots, n+n\}$,

(iii) $E=\left\{(i, n+j) \in N \times M: j \in A_{i}\left(R_{i}\right)\right.$ or $\left.j=i\right\}$,

(iv) for all $i \in N$ and each edge $(i, n+j) \in E$ where $j \in A_{i}\left(R_{i}\right)$ we set $u(i, n+j)=\bar{t}_{i j}$ and $u(i, n+i)=t_{i}$, and

(v) for all $i \in N$, we set $q(i)=t_{i}$.

Example 1. Let $N=\{1,2,3,4\}, t_{1}=t_{2}=1$ and $t_{3}=t_{4}=2$. Let $R \in \tilde{\mathcal{R}}$ be such that $A_{1}\left(R_{1}\right)=A_{2}\left(R_{2}\right)=\{3,4\}\left(\right.$ with $\left.\bar{t}_{13}=\bar{t}_{14}=\bar{t}_{23}=\bar{t}_{24}=1\right)$ and $A_{3}\left(R_{3}\right)=A_{4}\left(R_{4}\right)=\{1,2\}$ (with $\bar{t}_{31}=\bar{t}_{32}=\bar{t}_{41}=\bar{t}_{42}=2$ ). The constructed graph $g$ is depicted in Figure 1 .

The interpretation of the graph $g$ is that the agents in $M$ should be regarded as copies of the agents in $N$ and in particular, agent $n+i \in M$ is the copy of agent $i \in N$. Furthermore, agents $i \in N$ and $n+j \in M$ are connected by an edge if and only if agent $j$ is acceptable for agent $i$ or if $j=i$. A matching $x$ specifies for each $(i, l) \in E$ a non-negative integer $x_{i l} \in \mathbb{N}_{0}$. Any matching $x$ is equivalent to an allocation in the usual sense: $x_{i i}=x_{i(n+i)}, x_{i j}=x_{i(n+j)}$ for all $j \in A_{i}\left(R_{i}\right)$, and $x_{i j}=0$ for all $j \in U_{i}\left(R_{i}\right)$. Because an allocation will be defined by the matching values $x_{i l}$ on the edges, our construction guarantees that agent $n+j \in M$ can only provide goods to an agent $i \in N$ if agent $i$ finds the good provided by agent $j$ acceptable or if agent $j$ is his own copy. Finally, the upper bound on the matching value from agent $n+j$ to agent $i$ where $j \in A_{i}\left(R_{i}\right)$ is equal to the upper bound that represents the number of goods agent $i$ at most would like to receive from agent $j$. 
Recall that the balancedness conditions (1) and (2) must hold for any allocation. In the language of matching problems, this means that the required matching value is dictated by equations (1) and (2) which must be reformulated for the bipartite setting as follows:

$$
\begin{gathered}
\sum_{j \in A_{i}\left(R_{i}\right) \cup\{i\}} x_{i(n+j)}=q(i) \text { for all } i \in N, \\
\sum_{i \in A_{j}\left(R_{j}\right) \cup\{i\}} x_{j(n+i)}=q(i) \text { for all } i \in N .
\end{gathered}
$$

These conditions are fulfilled by any matching, because they express the perfectness condition in the matching instance.

A natural interpretation of the bipartite graph is therefore that agents in $M$ supply goods to the demanding agents in $N$. To obtain a maximal outcome, it is important to prevent matchings between agents in $N$ and their own copies in $M$ whenever there are other feasible flows or, equivalently, to prevent agents from supplying goods to their own copies whenever it is feasible to supply goods to other distinct agents (by the balancedness conditions, any agent supplying goods to other agents also receives equally many goods in return from acceptable agents). This can be achieved by introducing an artificial weight whenever agents supply their own goods to themselves. Let, for this purpose, $w_{i l}$ denote the weight associated when agent $l \in M$ supplies goods to agent $i$, and let, in particular, for each $(i, l) \in E$ :

$$
w_{i l}= \begin{cases}-1 & \text { if } l=n+i \\ 0 & \text { otherwise }\end{cases}
$$

For a given profile $R \in \tilde{\mathcal{R}}$, a given graph $g=(N, M, E, u, q)$ and given weights $w=\left(w_{i l}\right)_{(i, l) \in E}$, the (artificial) weight is maximized at any allocation $x \in \mathcal{F}$ that solves the following maximization problem:

$$
\max \sum_{(i, l) \in E} w_{i l} x_{i l} \text { s.t. conditions (1'), (2'), } x_{i l} \in \mathbb{N}_{0} \text { and } x_{i l} \leq u(i, l) \text { for all }(i, l) \in E \text {. }
$$

An allocation $x \in \mathcal{F}$ is a maximizer if it is a solution of the maximization problem (5). Let $\mathcal{V}(R, w) \subseteq$ $\mathcal{F}$ denote the set of all maximizers at profile $R \in \tilde{\mathcal{R}}$ for given weights $w=\left(w_{i l}\right)_{(i, l) \in E}$. For notational 
convenience, the value of an allocation $x$ at weight $w$ is given by $V(x, w)=\sum_{(i, l) \in E} w_{i l} x_{i l}$.

Lemma 1. If allocation $x$ belongs to $\mathcal{V}(R, w)$ at profile $R \in \tilde{\mathcal{R}}$, then $x \in \mathcal{X}(R)$.

The set of maximizers $\mathcal{V}(R, w)$ is non-empty for any profile $R \in \tilde{\mathcal{R}}$ since $\mathcal{V}(R, w) \subseteq \mathcal{X}(R)$ and $\mathcal{X}(R)$ is non-empty and finite for all $R \in \tilde{\mathcal{R}}$. However, as stated above, agents need not be indifferent between all allocations in the set $\mathcal{V}(R, w)$ since $\mathcal{V}(R, w) \subseteq \mathcal{X}(R)$. Hence, in order to define a priority mechanism based on a solution to maximization problem (5), a refined selection from the set $\mathcal{V}(R, w)$ is necessary which will be based on the priority-ordering $\pi$.

We will modify the weights $w$ in order to take the priority-ordering $\pi$ into account. Let $\varepsilon_{0} \in(0,1)$ and $\varepsilon_{i-1}=\left(1+t_{i}\right) \varepsilon_{i}$ for each $i \in\{1, \ldots, n\}$. By construction of $\varepsilon_{i}$, it follows that: ${ }^{11}$

$$
1>\varepsilon_{0} \geq \varepsilon_{i}>\sum_{k=i+1}^{n} t_{k} \varepsilon_{k}>0 \text { for all } i \in\{0, \ldots n-1\} .
$$

To guarantee a larger allocation to agents with higher priorities, the weight associated with an edge in the matching instance will be monotonically increasing with higher priorities. More specifically, let for each $(i, l) \in E$ :

$$
\tilde{w}_{i l}= \begin{cases}-1 & \text { if } l=n+i \\ \varepsilon_{\pi(i)} & \text { otherwise. }\end{cases}
$$

The above construction means that the agent with the highest priority (i.e., the agent with $\pi(i)=$ 1) will receive the highest edge weight (for edges $(i, l) \in E \backslash\{(i, n+i)\}$ ), the agent with the secondhighest priority (i.e., the agent with $\pi(i)=2$ ) will receive the second-highest edge weight, and so on.

Our second main result demonstrates that a mechanism that selects an allocation from the set of maximizers for each profile in $\tilde{\mathcal{R}}$ and any given priority-ordering is a priority mechanism. From Theorem 1, it is already known that such a mechanism is non-manipulable on the domain $\tilde{\mathcal{R}}$.

Proposition 1. For a given priority-ordering $\pi$, a mechanism $\varphi$ selecting for each profile $R \in \tilde{\mathcal{R}}$ an allocation from $\mathcal{V}(R, \tilde{w})$ is a priority mechanism based on $\pi$.

\footnotetext{
${ }^{11}$ To see this, note that $\varepsilon_{n-1}=\left(1+t_{n}\right) \varepsilon_{n}>t_{n} \varepsilon_{n}$ since $\varepsilon_{n}>0$ and, consequently, $\varepsilon_{n-2}=\left(1+t_{n-1}\right) \varepsilon_{n-1}=$ $\varepsilon_{n-1}+t_{n-1} \varepsilon_{n-1}>t_{n} \varepsilon_{n}+t_{n-1} \varepsilon_{n-1}$. Condition (6) then follows by repeating these arguments.
} 


\section{Discussion and Extensions}

This section discusses essentially single-valued cores and random mechanisms under two separate headings. ${ }^{12}$ The section also contains a discussion of possible extensions of the model related to, e.g., more general preferences and trading networks.

\subsection{Essentially Single-Valued Cores}

Theorem 1 establishes that, in the considered exchange problem, there exist mechanisms which are individually rational, efficient, and non-manipulable on the domain $\tilde{\mathcal{R}}$. This is surprising as a number of previous impossibility results for the combination of these axioms have been established by applying an essentially single-valued cores result by Sönmez (1999). Below, we connect his result to the considered exchange problem.

Given $R \in \tilde{\mathcal{R}}$, the core of $R$, denoted by $\mathcal{C}(R)$, consists of all feasible allocations $x \in \mathcal{F}$ which are not dominated via some coalition and some allocation meaning that there exists no $\emptyset \neq S \subseteq N$ and $y \in \mathcal{F}$ such that (i) $y_{i} R_{i} x_{i}$ for all $i \in S$, (ii) $y_{j} P_{j} x_{j}$ for some $j \in S$ and (iii) $\left\{j \in N: y_{i j} \neq 0\right\} \subseteq S$ for all $i \in S$. The core of $R$ is essentially single-valued if for all $x, y \in \mathcal{C}(R)$ we have $x_{i} I_{i} y_{i}$ for all $i \in N$. Note that, if $\mathcal{C}(R)=\emptyset$, then the core of $R$ is essentially single-valued.

Let $\tilde{\mathcal{R}}^{1}$ denote the set of all profiles $R \in \tilde{\mathcal{R}}$ such that for all $i \in N$ and all $j \in A_{i}\left(R_{i}\right)$ we have $\bar{t}_{i j}=1$ and $t_{i}=1$ (i.e., any agent demands at most one unit of any acceptable good, and any agent supplies at most one unit of their goods). This corresponds to the classical dichotomous domain by Bogomolnaia and Moulin (2004). Then it is easy to check that the domain $\tilde{\mathcal{R}}^{1}$ satisfies Assumptions A and B of Sönmez (1999). ${ }^{13}$ Hence, his main result applies, which shows the following: if there exists an individually rational, efficient, and non-manipulable mechanism, then for any profile where the core is non-empty we have (a) the core is essentially single-valued and (b) the mechanism chooses a core allocation. However, here for any $R \in \tilde{\mathcal{R}}^{1}$, if the core of $R$ is non-empty, then the set of individually rational and efficient allocations is essentially single-valued (and the core is essentially single-valued). ${ }^{14}$ But then any priority mechanism chooses a core allocation. Note that

\footnotetext{
${ }^{12} \mathrm{We}$ are grateful to the referees and the Co-Editor for bringing our attention to random mechanisms.

${ }^{13}$ In our framework (without externalities), Assumption A says that for any allocation $x$ we have $x_{i} I_{i} \omega_{i}$ if and only if $x_{i}=\omega_{i}$ and Assumption B says that whenever for two allocations $x$ and $y$ with $x_{i} P_{i} y_{i}$ and $x_{i} R_{i} \omega_{i}$, there exists a preference relation $R_{i}^{\prime}$ such that $x_{i} R_{i}^{\prime} \omega_{i} R_{i}^{\prime} y_{i}$.

${ }^{14}$ Note that for any $R \in \tilde{\mathcal{R}}^{1}$, if the set of individually rational and efficient allocations is not essentially single-valued, then any two individually rational and efficient allocations, which are not regarded indifferent by all agents, dominate (via some coalition) each other and the core must be empty: more formally, for $R \in \tilde{\mathcal{R}}$ and any two individually rational and efficient allocations $x$ and $y$ for which not $x_{i} I_{i} y_{i}$ for all $i \in N$, for $S=\left\{i \in N: x_{i i}=0\right\}$ we have for all $i \in S, x_{i} R_{i} y_{i}$,
} 
Proposition 1 of Sönmez (1999) shows that, when the core of each profile is externally stable, then any selection from the core correspondence is non-manipulable. ${ }^{15}$ External stability implies that the core is non-empty for any profile, but here, if the core is non-empty, then the set of individually rational and efficient allocations is essentially single-valued. As this is often not the case, the core is often empty and Proposition 1 of Sönmez (1999) cannot be used to show the non-manipulability of priority mechanisms.

Once non-unitary endowments are allowed, the domain $\tilde{\mathcal{R}}$ does not satisfy Assumption B of Sönmez (1999). This is illustrated in the next example.

Example 2. We use the instance introduced in Example 1, i.e., $N=\{1,2,3,4\}, t_{1}=t_{2}=1$, $t_{3}=t_{4}=2$, and $R \in \tilde{\mathcal{R}}$ is such that $A_{1}\left(R_{1}\right)=A_{2}\left(R_{2}\right)=\{3,4\}\left(\right.$ with $\bar{t}_{13}=\bar{t}_{14}=\bar{t}_{23}=\bar{t}_{24}=1$ ) and $A_{3}\left(R_{3}\right)=A_{4}\left(R_{4}\right)=\{1,2\}$ (with $\bar{t}_{31}=\bar{t}_{32}=\bar{t}_{41}=\bar{t}_{42}=2$ ). If agent 3 comes before agent 4 in the priority-order $\pi$, then $(3,3,12,0)$ is the unique $\pi$-priority allocation (where this stands for agent 1 receiving one unit from agent 3, agent 2 receiving one unit from agent 3, agent 3 receiving one unit from both agent 1 and agent 2, and agent 4 keeping his endowment). If agent 4 comes before agent 3 in the priority-order $\pi$, then $(4,4,0,12)$ is the unique $\pi$-priority allocation. Note that $(3,3,12,0) P_{3}(3,4,1,2) P_{3} \omega_{3}$ but there exists no $R_{3}^{\prime}$ such that $(3,3,12,0) P_{3}^{\prime} \omega_{3} P_{3}^{\prime}(3,4,1,2)$. The latter conclusion follows since $(3,3,12,0) P_{3}^{\prime} \omega_{3}$ implies $1 \in A_{3}\left(R_{3}^{\prime}\right)$ and $\bar{t}_{31}^{\prime} \geq 1$, and thus $(3,4,1,2) P_{3}^{\prime} \omega_{3}$. Hence, Assumption B is violated for the domain $\tilde{\mathcal{R}}$ and, at the same time, any priority mechanism is individually rational, efficient, and non-manipulable.

The above example also shows that, in general, we do not have dichotomous preferences in the domain $\tilde{\mathcal{R}}$. We may have many distinct indifference classes for preferences in the domain $\tilde{\mathcal{R}}$ and yet, by Theorem 1, there exists an individually rational, efficient, and non-manipulable mechanism.

Finally, it is demonstrated that a priority mechanism with the same order may select different allocations when choosing from the set of individually rational and efficient allocations (as in Manjunath and Westkamp, 2019).

Example 3. Let $N=\{1,2,3,4\}$ and $t_{1}=t_{2}=t_{3}=t_{4}=1$. Let $R \in \tilde{\mathcal{R}}$ be such that $A_{1}\left(R_{1}\right)=\{2\}$, $A_{2}\left(R_{2}\right)=\{3\}, A_{3}\left(R_{3}\right)=\{1,4\}$, and $A_{4}\left(R_{4}\right)=\{3\}\left(\right.$ with $\left.\bar{t}_{12}=\bar{t}_{23}=\bar{t}_{31}=\bar{t}_{34}=\bar{t}_{43}=1\right)$. Then $\mathcal{X}(R)=\{(2,3,1,0)\}$, i.e., there is a unique individually rational and maximal allocation which and for some $j \in S, x_{j} P_{j} y_{j}$, i.e., $x$ dominates $y$ with the coalition $S$ (and the same argument applies for $y$ in the role of $x$ and $x$ in the role of $y$ ). Thus, the core of $R$ is empty.

${ }^{15}$ See also Demange (1987) for an important study of non-manipulable cores. 
is chosen by any priority mechanism. However, the allocation $(0,0,4,3)$ is individually rational and efficient which is selected by any priority mechanism which chooses from the whole set of individually rational and efficient allocations and where agent 4 occupies the first position in the priority-order (and such a priority mechanism would not necessarily result in a maximal allocation). Note that the same argument applies if a priority mechanism chooses from the set of all feasible allocations.

\subsection{Random Mechanisms}

Priority mechanisms are unfair in the sense that the agent in the first position of the priority-ordering receives, for any profile $R$, his most-preferred bundle among all allocations in $\mathcal{X}(R)$ (but this is not the case for the agent in last position of the priority-ordering). To establish fairness, one may consider random allocations and random mechanisms, which we define briefly below.

A random allocation for $R$ is a probability distribution $p$ over $\mathcal{F}$. For all $x \in \mathcal{F}$, let $p(x)$ denote the probability of allocation $x$. The support of $p$ is given by the allocations which are chosen with positive probability by $p$, i.e. $\operatorname{supp}(p)=\{x \in \mathcal{F}: p(x)>0\}$. Then $p$ is ex-post individually rational for $R$ if for all $x \in \operatorname{supp}(p), x$ is individually rational. Analogously, ex-post maximality and ex-post efficiency are defined. For two random allocations $p$ and $q$, we say that $p$ stochastically $R_{i}$-dominates $q$ (where we write equivalently $p_{i}$ stochastically $R_{i}$-dominates $q_{i}$ ), denoted by $p_{i} R_{i}^{s d} q_{i}$, if for all $y \in \mathcal{F}$ we have:

$$
\sum_{x \in \mathcal{F}: x_{i} R_{i} y_{i}} p(x) \geq \sum_{x \in \mathcal{F}: x_{i} R_{i} y_{i}} q(x)
$$

Then $p_{i} P_{i}^{s d} q_{i}$ if $p_{i} R_{i}^{s d} q_{i}$ and not $q_{i} R_{i}^{s d} p_{i}$. A random mechanism $\phi$ chooses for any profile $R \in \tilde{\mathcal{R}}$ a random allocation for $R$. The random mechanism $\phi$ is ex-post individually rational if, for any profile $R$, the random allocation is ex-post individually rational for $R$. Analogously, ex-post maximality and ex-post efficiency are defined for random mechanisms.

Let now $\varphi^{\pi}$ denote a deterministic priority mechanism using $\pi$ as a priority-ordering and $\Pi$ denote the set of all priority-orderings. Then let $R P=\sum_{\pi \in \Pi} \frac{1}{n !} \varphi^{\pi}$ denote the random priority mechanism putting equal priority on each priority-ordering. Because deterministic priority mechanisms are individually rational, maximal and efficient, the random priority mechanism is ex-post individually rational, ex-post maximal and ex-post efficient.

For random mechanisms, axioms are often defined in terms of stochastic dominance. The random 
mechanism is sd-non-manipulable if, for all $R, R^{\prime} \in \tilde{\mathcal{R}}$ such that $R^{\prime}=\left(R_{i}^{\prime}, R_{-i}\right)$ for some $i \in N$, we have $\phi_{i}(R) R_{i}^{s d} \phi_{i}\left(R^{\prime}\right)$. The random mechanism is sd-efficient if, for all $R \in \tilde{\mathcal{R}}$, there exists no random allocation $p$ for $R$ such that $p_{i} R_{i}^{s d} \phi_{i}(R)$ for all $i \in N$ and $p_{j} P_{i}^{s d} \phi_{j}(R)$ for some $j \in N$.

For a random allocation $p$ and agent $j$, let $\left[p_{j}\right]^{-j j}$ denote the probabilities which $p_{j}$ induces on any $x_{j l} \in \mathbb{N}_{0}$ with $j \neq l$. This takes into account that we only consider individual rational allocations, since all probabilities in $\left[p_{j}\right]^{-j j}$ are zero for allocations involving agent $j$ sending a service to herself. The random mechanism is sd-fair (subject to individual rationality) if for all $R \in \tilde{\mathcal{R}}$ and all $i, j \in N$, $\phi_{i}(R) R_{i}^{s d}\left[\phi_{j}(R)\right]^{-j j}$.

Now, from our results we obtain the following corollary.

Corollary 1. The random priority mechanism is sd-non-manipulable, sd-efficient and sd-fair.

In Corollary 1, sd-non-manipulability and sd-fairness are quite obvious, whereas sd-efficiency is more surprising and relies on the fact that preferences are dichotomous over single agents (see also Bogomolnaia and Moulin, 2004). ${ }^{16}$ Besides random priority mechanisms, it would be interesting whether there are any other "nice" random mechanisms which are not simply a mixing of deterministic mechanisms. This question is left for future research.

\subsection{Extensions}

This section contains discusses three possible extensions of the considered model. ${ }^{17}$

\subsubsection{More General Preferences}

One may argue that the upper bounds on how many units of the good agent $i$ at most would like to receive from agent $j$ is extreme in the following sense. Suppose that there are two agents, called 1 and 2 , such that $t_{1}=t_{2}=3$. Now, if for profile $R$ we have $\bar{t}_{12}=2$, then $(22,11) P_{1} w_{1} P_{1}(222,111)$ meaning that agent 1 would strictly prefer his endowment to receiving three units of the good from agent 2. One may argue that agent 1 has a preference such that $(22,11) P_{1}(222,111) P_{1} w_{1}$, i.e., receiving two units of the good from agent 2 is optimal, but receiving three units is still better than his endowment. This would correspond to agent 1 having a "peak" at two units and a maximum at three units.

\footnotetext{
${ }^{16}$ This is not true in general. Bogomolnaia and Moulin (2001) show that ex-post efficiency (or ex-post maximality) on the strict domain does not imply sd-efficiency when agents have unitary endowments.

${ }^{17}$ We thank the referees and the Co-Editor for suggesting these extensions.
} 
It is easy to see that including such preferences would result in a manipulable mechanism (if the mechanism is efficient and individually rational). Suppose, for instance, that agent 2 has the following preference $(222,111) P_{2}(22,11) P_{2} w_{2}$. Then $x=(222,111)$ and $x^{\prime}=(22,11)$ are the two individual rational and efficient allocations at the preference profile $\left(P_{1}, P_{2}\right)$. Thus, any mechanism that chooses an individual rational and efficient allocation must choose either $x$ or $x^{\prime}$. Suppose now that allocation $x$ is chosen. Then agent 1 can report $P_{1}^{\prime}$ as $(22,11) P_{1}^{\prime} w_{1} P_{1}^{\prime}(222,111)$ and the mechanism must choose allocation $x^{\prime}$, since this is the only individual rational and efficient allocation at $\left(P_{1}^{\prime}, P_{2}\right)$. This is a successful manipulation by agent 1 . A symmetric argument, where agent 2 can manipulate, can be made if allocation $x^{\prime}$ is chosen. This impossibility is not surprising; see, for instance Konishi et al. (2001) where agents are endowed with multiple types of indivisible goods and have more general preferences.

Another possibility is that agents possess more general preferences but are only allowed to report

profiles belonging to $\tilde{\mathcal{R}}$ to the priority mechanism. Then non-manipulability becomes meaningless as agents cannot report their true preferences and one would have to consider games induced by the mechanism and the general preferences. For instance, if all agents report that no goods are acceptable, then this is a Nash equilibrium outcome which is in general neither efficient nor maximal for the true preferences.

\subsubsection{Non-Integer Endowments and Upper Bounds}

Our analysis can easily accommodate endowments and upper bounds given by rational numbers. Without going into detail, for any profile $R$ (where for any $i \in N, t_{i}$ and $\bar{t}_{i j}$ are rational numbers), let $d$ denote the greatest common denominator of all constraints. Then, for the profile $R$ in terms of unit $d$, agent $i$ is endowed with $d t_{i}$ units of his own good, and $d \bar{t}_{i j}$ represents agent $i$ 's upper bound for goods from agent $j$. Our construction applies to the profile $R$ in terms of unit $d$ to obtain a priority allocation which is individually rational and maximal. For non-manipulability, for two profiles $R$ and $R^{\prime}$, let $R$ be in terms of unit $d$ and $R^{\prime}$ in terms of unit $d^{\prime}$. However, we can then express $R$ in terms of unit $d d^{\prime}$ and the priority allocation for $R$ in terms of unit $d$ is also a priority allocation in terms of unit $d d^{\prime}$. Similarly, we can express $R^{\prime}$ in terms of unit $d d^{\prime}$, and the priority allocation for $R^{\prime}$ is also a priority allocation in terms of unit $d d^{\prime}$. Because the same priority ordering is used, non-manipulability follows from Theorem 1 . Moreover, the algorithm computing a maximum weight 
circulation is strongly polynomial (Orlin, 1993); thus, the running time does not depend on $d$ or $d^{\prime} .{ }^{18}$

\subsubsection{Embedding in Trading Networks}

It is next demonstrated that our model can be embedded in the general framework of trading networks by Kominers et al. (2020).

In any allocation $x$, for any $i, j \in N$, the number $x_{i j}$ can be viewed as a contract between agent $i$ and agent $j$, where agent $j$ provides $x_{i j}$ units of his own good to agent $i$. For later purposes, the number $x_{i j}$ is decomposed into separate units $1_{i j}, 2_{i j}, \ldots, x_{i j}$ where $k_{i j}$ stands for the $k$ th unit of the good provided by agent $j$ to agent $i$. Then agent $i$ is the buyer in contract $k_{i j}$ and agent $j$ is the seller in contract $k_{i j}$. We write $i=b\left(k_{i j}\right)$ and $j=s\left(k_{i j}\right)$. Prices are ignored and are all implicitly set to one. The set of all contracts is denoted by:

$$
\mathcal{Y}=\left\{k_{i j}: i, j \in N \text { with } i \neq j \text { and } k \in\left\{1, \ldots, t_{j}\right\}\right\}
$$

An allocation is then simply a subset of contracts $Y \subseteq \mathcal{Y}$. Let $Y_{\rightarrow i}=\{y \in Y: b(y)=i\}$ denote the set of contracts in $Y$ where agent $i$ is a buyer and $Y_{i \rightarrow}=\{y \in Y: s(y)=i\}$ the set of contracts in $Y$ where agent $i$ is a seller. Let $Y_{i j}=Y_{\rightarrow i} \cap Y_{j \rightarrow}$ denote the set of contracts in $Y$ where agent $i$ is the

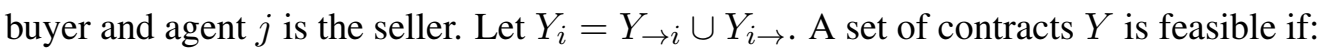

(i) for all $i \in N,\left|Y_{\rightarrow i}\right|=\left|Y_{i \rightarrow}\right| \leq t_{i}$,

(ii) for all $i, j \in N$, if $k_{i j} \in Y$ and $k>1$, then $(k-1)_{i j} \in Y$, and;

(iii) for all $i, j \in N$, if $k_{j i} \in Y$ and $k>1$, then $(k-1)_{j i} \in Y$.

Note that condition (i) corresponds to equations (1) and (2), and condition (ii) says that if agent $j$ provides to agent $i$ the $k$ th unit of the good, then agent $j$ provides to agent $i$ the $k-1$ th unit of the good (and similarly for condition (iii)). We say that $Y_{i}$ is feasible for agent $i$ if (i), (ii) and (iii) hold for agent $i$. Note that any feasible allocation $Y$ corresponds to an allocation $y$ in the original model by setting for all $i, j \in N$ with $i \neq j, y_{i j}=\left|Y_{i j}\right|$ and $y_{i i}=t_{i}-\left|Y_{i \rightarrow \mid}\right|$ (and vice versa as above).

Given profile $R \in \tilde{\mathcal{R}}$, agent $i$ 's utility function over subsets of contracts $Y \subseteq \mathcal{Y}_{i}$ which are feasible for agent $i$ is given by (i) $U_{i}(Y)=\left|Y_{i \rightarrow \mid}\right|$ if $Y_{i j}=\emptyset$ for all $j \in U_{i}\left(R_{i}\right)$ and $\left|Y_{i j}\right| \leq \bar{t}_{i j}$ for

\footnotetext{
${ }^{18}$ We leave the incorporation of irrational constraints for future research.
} 
all $j \in A\left(R_{i}\right)$, and (ii) $U_{i}(Y)=-\infty$ otherwise. Then an allocation $Y$ is feasible if for all $i \in N$, $U_{i}(Y) \neq-\infty$. Note that $U_{i}(\emptyset)=0$.

Then agent $i$ 's choice correspondence for subsets of contracts is defined as follows. For all $Y \subseteq$ $\mathcal{Y}_{i}$, let:

$$
C_{i}(Y)=\left\{X \subseteq Y: X \text { is feasible for } i \text { and } U_{i}(X) \geq U_{i}\left(X^{\prime}\right) \text { for all } X^{\prime} \subseteq Y \text { feasible for } i\right\}
$$

We then write for $Y \subseteq \mathcal{Y}, C_{i}(Y)=C_{i}\left(Y_{i}\right)$.

Then allocation $Y$ is individually rational if for all $i \in N, Y_{i} \in C_{i}(Y)$. The allocation $Y$ is maximal if $Y$ is individually rational and there exists no other individually rational allocation $W$ such

that $\sum_{i \in N} U_{i}(W)>\sum_{i \in N} U_{i}(Y)$. Then allocation $Y$ is stable if $Y$ is individually rational and there exists no $Z \subseteq \mathcal{Y} \backslash Y$ such that for all $i \in N(Z)=\left\{i \in N: Z_{i} \neq \emptyset\right\}$ and all $W \in C_{i}(Y \cup Z)$, $Z_{i} \subseteq W_{i}$.

Corollary 2. If $Y$ is individually rational and maximal, then $Y$ is stable.

Thus, our results establish that there exists a stable and non-manipulable mechanism. Again, this is surprising as there often does not exist any stable and non-manipulable mechanism.

Below, we verify that agents' preferences satisfy monotone substitutability. Hence, by Theorem 1 of Kominers et al. (2020), stability is equivalent to "chain stability." Moreover, one may verify in Example 3 that $(0,0,4,3)$ is stable, and the set of individually rational and maximal allocations is in general a strict subset of the set of stable allocations.

Then agent $i$ 's choice function is monotone substitutable if (1) for all $Y, Z \subseteq \mathcal{Y}_{i}$ such that $Y_{i \rightarrow}=$ $Z_{i \rightarrow}$ and $Y_{\rightarrow i} \subseteq Z_{\rightarrow i}$, for every $Y^{*} \in C_{i}(Y)$ there exists $Z^{*} \in C_{i}(Z)$ such that (i) $Y_{i \rightarrow}^{*} \subseteq Z_{i \rightarrow}^{*}$, (ii) $\left(Y_{\rightarrow i} \backslash Y_{\rightarrow i}^{*}\right) \subseteq\left(Z_{\rightarrow i} \backslash Z_{\rightarrow i}^{*}\right)$, and (iii) $\left|Z_{\rightarrow i}^{*}\right|-\left|Z_{i \rightarrow}^{*}\right| \geq\left|Y_{\rightarrow i}^{*}\right|-\left|Y_{i \rightarrow}^{*}\right|$; and (2) for all $Y, Z \subseteq \mathcal{Y}_{i}$ such that $Y_{\rightarrow i}=Z_{\rightarrow i}$ and $Y_{i \rightarrow} \subseteq Z_{i \rightarrow}$, for every $Y^{*} \in C_{i}(Y)$ there exists $Z^{*} \in C_{i}(Z)$ such that (i)

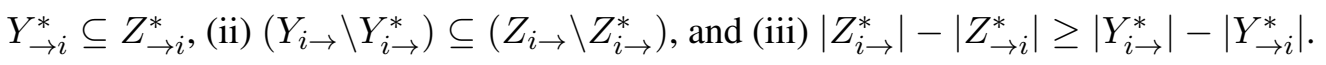

Lemma 2. For all $i \in N$ and all $R \in \tilde{\mathcal{R}}$, agent $i$ 's choice function $C_{i}$ is monotone substitutable.

\section{Concluding Remarks}

This paper has modeled time exchanges as matching markets. On a restricted, yet natural preference domain, it has been demonstrated that a priority mechanism can be formulated as a max-weight 
matching problem and, furthermore, that such a mechanism is non-manipulable and always makes a selection from the set of individually rational, efficient, and balanced allocations. No mechanism with these properties exists in the general preference domain (Sönmez, 1999, Corollary 1).

Even if the considered priority mechanism has been demonstrated to satisfy all properties of interest on a restricted preference domain, the mechanism can be criticized from a fairness perspective as it discriminates low-priority agents (see the discussion in Section 6.2). For this reason, it is important to characterize the entire class of mechanisms that satisfies the axioms of interest to see if such discrimination can be avoided or not. Moreover, even though the considered domain restriction is natural for many time exchanges, it may also be of importance to find a maximal domain result where the above mentioned impossibility can be escaped. Both these open problems are left for future research.

Finally, we would again like to point out that, even if the timebanking problem was introduced in Section 2, all results stated in this paper can be adopted on other types of time exchanges. Consequently, the proposed mechanism is not restricted to the timebanking application (see also the Introduction for additional examples). ${ }^{19}$

\section{Appendix A: Proofs}

Appendix A contains the proofs of all results except Theorem 1, which is in Appendix B.

Proof of Lemma 1. Suppose that allocation $x$ belongs to $\mathcal{V}(R, w)$. The fact that $x$ is feasible and individually rational follows directly from the construction of the graph $g=(N, M, E, u, q)$ and by definition of the maximization problem (5), i.e., agent $n+j \in M$ is only connected to an agent $i \in N$ if agent $j \in A_{i}\left(R_{i}\right) \cup\{i\}$, non-zero matching values are between connected agents and the matching value never exceeds the upper bounds $\bar{t}_{i j}$ on any edge $(i, n+j) \in E$.

To show that allocation $x$ is maximal, it will be demonstrated that $x$ minimizes the total matching

\footnotetext{
${ }^{19}$ Another problem that recently has been solved, using a version of the priority mechanism proposed in this paper, is the "seminar exchange problem." This problem was initiated in Scandinavia in the fall of 2018 (by one of the authors of this paper) to help final year $\mathrm{PhD}$ students to practice their job market talks at external departments. To solve this problem, Economics departments classified 11 different research field as acceptable and unacceptable. Job market candidates, on the other hand, classified themselves by one of the 11 different research fields and all departments as either acceptable or unacceptable. A specific construction guaranteed that the job market candidates also played the role of their departments and could, therefore, be engaged in time-balanced seminar exchanges with other students. The specific construction made it possible to apply our model and mechanism in this setting, due to limited space we will not explain further details but are happy to answer any questions and provide further details via email. In total, 10 departments and 21 job market candidates from Denmark, Norway, and Sweden participated in the centralized market for seminar exchange. In the end, all candidates were matched to some department in a balanced sense (i.e., each department organized exactly as many seminars as their own students were invited to).

All details of the seminar exchange market are described at the Swedish Economics blog Ekonomistas. See https://ekonomistas.se/2018/12/03/en-skandinavisk-matchningsmarknad-for-doktorandseminarier/.
} 
value between agents $i \in N$ and their respective clones $i+n \in M$. Because $x \in \mathcal{V}(R, w)$ is a maximizer, it follows that:

$$
\sum_{(i, l) \in E} w_{i l} x_{i l} \geq \sum_{(i, l) \in E} w_{i l} x_{i l}^{\prime} \text { for any feasible allocation } x^{\prime} \text { in program (5). }
$$

Given the construction of the costs in condition (4), it now follows from condition (7) that:

$$
\sum_{i=1}^{n} w_{i(n+i)} x_{i(n+i)} \geq \sum_{i=1}^{n} w_{i(n+i)} x_{i(n+i)}^{\prime} .
$$

Because $w_{i(i+n)}=-1$ for all $i \in N$, by condition (4), the above inequality can be rewritten as:

$$
\sum_{i=1}^{n} x_{i(n+i)}^{\prime} \geq \sum_{i=1}^{n} x_{i(n+i)}
$$

But this condition means that allocation $x$ minimizes the total matching value between agents $i \in N$ and their respective clones $i+n \in M$ among all feasible allocations, which is the desired conclusion.

Proof of Proposition 1. It is first demonstrated that $\mathcal{V}(R, \tilde{w}) \subseteq \mathcal{V}(R, w)$ for each profile $R \in \tilde{\mathcal{R}}$. Suppose now that $x \in \mathcal{V}(R, w)$ but $x^{\prime} \notin \mathcal{V}(R, w)$ for some $x^{\prime}$ that is feasible in the optimization program defined in (5). To reach the conclusion, it is sufficient to show $x^{\prime} \notin \mathcal{V}(R, \tilde{w})$.

Note that $x \in \mathcal{V}(R, w)$ and $x^{\prime} \notin \mathcal{V}(R, w)$ imply $V(x, w)>V\left(x^{\prime}, w\right)$. This conclusion together with $w_{i l} \in\{-1,0\}$ and $x_{i l} \in \mathbb{N}_{0}$ for all $(i, l) \in E$ and $\varepsilon_{0}<1$ gives $V(x, w)>V\left(x^{\prime}, w\right)+\varepsilon_{0}$. Because $\tilde{w}_{i l} \geq w_{i l}$ for all $(i, l) \in E$ by construction, it holds that $V(x, \tilde{w}) \geq V(x, w)$. This, together with the above inequalities, imply $V(x, \tilde{w})>V\left(x^{\prime}, w\right)+\varepsilon_{0}$. To complete this part of the proof, we show that $V\left(x^{\prime}, w\right)+\varepsilon_{0} \geq V\left(x^{\prime}, \tilde{w}\right)$, since this condition together with the above conclusions then show $V(x, \tilde{w})>V\left(x^{\prime}, \tilde{w}\right)$, i.e., that $x^{\prime} \notin \mathcal{V}(R, \tilde{w})$.

To demonstrate $V\left(x^{\prime}, w\right)+\varepsilon_{0} \geq V\left(x^{\prime}, \tilde{w}\right)$, we partition $E$ into two disjoint sets, $E^{1}$ and $E^{2}$, where the former set contains all edges $(i, l)$ in $E$ where $l \neq i+n$ and the latter contains all edges $(i, l)$ in $E$ where $l=i+n$. Consequently, $w_{i l}=0<\tilde{w}_{i l}=\varepsilon_{i}$ for all $(i, l) \in E^{1}$ and $w_{i l}=\tilde{w}_{i l}=-1$ 
for all $(i, l) \in E^{2}$. Hence, the inequality $V\left(x^{\prime}, w\right)+\varepsilon_{0} \geq V\left(x^{\prime}, \tilde{w}\right)$ can be rewritten as:

$$
\begin{aligned}
V\left(x^{\prime}, w\right)+\varepsilon_{0} & =\sum_{(i, l) \in E} w_{i l} x_{i l}^{\prime}+\varepsilon_{0}, \\
& =\sum_{(i, l) \in E^{1}} w_{i l} x_{i l}^{\prime}+\sum_{(i, l) \in E^{2}} w_{i l} x_{i l}^{\prime}+\varepsilon_{0}, \\
& =\sum_{(i, l) \in E^{2}} \tilde{w}_{i l} x_{i l}^{\prime}+\varepsilon_{0}, \\
& \geq \sum_{(i, l) \in E} \tilde{w}_{i l} x_{i l}^{\prime} \\
& =\sum_{(i, l) \in E^{1}} \tilde{w}_{i l} x_{i l}^{\prime}+\sum_{(i, l) \in E^{2}} \tilde{w}_{i l} x_{i l}^{\prime}, \\
& =\sum_{(i, l) \in E^{1}} \varepsilon_{i} x_{i l}^{\prime}+\sum_{(i, l) \in E^{2}} \tilde{w}_{i l} x_{i l}^{\prime}, \\
& =V\left(x^{\prime}, \tilde{w}\right) .
\end{aligned}
$$

or, equivalently, as:

$$
\varepsilon_{0} \geq \sum_{(i, l) \in E^{1}} \varepsilon_{i} x_{i l}^{\prime}
$$

Conditions (6) and (1') together with the fact that $\varepsilon_{i} x_{i l} \geq 0$ for all $(i, l) \in N \times M$ now give:

$$
\varepsilon_{0}>\sum_{i \in N} \varepsilon_{i} t_{i} \geq \sum_{(i, l) \in E^{1}} \varepsilon_{i} x_{i l}^{\prime}
$$

But then condition (8) must hold. Hence, $\mathcal{V}(R, \tilde{w}) \subseteq \mathcal{V}(R, w)$. Thus, by Lemma $1 \varphi(R) \in \mathcal{X}(R)$.

To conclude the proof, it needs only to be demonstrated that $\varphi$ is a priority mechanism. But this follows directly from the construction of the weights $\varepsilon_{i}$. To see this, recall from condition (6) that $\varepsilon_{i}>\sum_{k=i+1}^{n} t_{k} \varepsilon_{k}$ for all $i \in\{1, \ldots, n-1\}$. Hence, assigning one additional unit to agent $i$ in maximization problem (5) is strictly preferred to assigning $t_{j}$ units to each agent $j \in N$ with $\pi(i)<\pi(j)$. Thus, $\mathcal{V}(R, \tilde{w})$ is a selection from $\mathcal{V}(R, w) \subseteq \mathcal{X}(R)$ that first maximizes the number of goods that agent $i_{1} \in N$ with $\pi\left(i_{1}\right)=1$ receives from acceptable agents (i.e., a selection from the set $\mathcal{Z}^{i_{1}, \mathcal{V}(R, w)}(R)$ ), and then maximizes the number of goods that agent $i_{2} \in N$ with $\pi\left(i_{1}\right)=2$ receives from acceptable agents (i.e., a selection from the set $\mathcal{Z}^{i_{2}, i_{1}}(R)$ ), and so on. This is the definition of a priority mechanism. 
Proof of Corollary 1. Because any deterministic priority mechanism $\varphi^{\pi}$ is non-manipulable, for all $R, R^{\prime} \in \tilde{\mathcal{R}}$ such that $R^{\prime}=\left(R_{i}^{\prime}, R_{-i}\right)$ for some $i \in N$, we have $\varphi_{i}^{\pi}(R) R_{i} \varphi_{i}^{\pi}\left(R^{\prime}\right)$. But then the random priority mechanism is sd-non-manipulable.

For sd-efficiency, note that $\phi(R)$ is ex-post maximal, and, for all $x, y \in \operatorname{supp}(\phi(R))$, we have:

$$
\sum_{i \in N} \sum_{j \in A_{i}\left(R_{i}\right)} x_{i j}=\sum_{i \in N} \sum_{j \in A_{i}\left(R_{i}\right)} y_{i j} \equiv m
$$

Now, if for some random allocation $p$ for $R$ we have $p_{i} R_{i}^{s d} \phi_{i}(R)$ for all $i \in N$ and $p_{j} P_{i}^{s d} \phi_{j}(R)$ for some $j \in N$, then $p$ is ex-post individually rational. But then it follows that:

$$
\sum_{i \in N} \sum_{x \in \operatorname{supp}(p)} \sum_{j \in A_{i}\left(R_{i}\right)} x_{i j}>m
$$

But then there must exist $y \in \operatorname{supp}(p)$ such that $\sum_{i \in N} \sum_{j \in A_{i}\left(R_{i}\right)} y_{i j}>m$, which is a contradiction to ex-post maximality of $\phi(R)$. Thus, $\phi$ is sd-efficient.

For sd-fairness, note that for any $i, j \in N$, the probability that $i$ occupies the first position in a priority-ordering is equal to the probability that $j$ occupies the first position in a priority-ordering. Similarly, for any $l \in N \backslash\{i, j\}$, the probability that $i$ occupies the second position after $l$ in a priorityordering is equal to the probability that $j$ occupies the second position after $l$ in a priority-ordering, and so on. But then it follows that the random priority mechanism is sd-fair.

Proof of Corollary 2. Suppose that $Y$ is not stable. As $Y$ is individually rational, there exists $Z \subseteq \mathcal{Y} \backslash Y$ such that for all $i \in N(Z)=\left\{i \in N: Z_{i} \neq \emptyset\right\}$ and all $W \in C_{i}(Y \cup Z), Z_{i} \subseteq W_{i}$. But then for all $i \in N(Z), Y_{i} \notin C_{i}(Y \cup Z)$ and $U_{i}(Y \cup Z)>U_{i}(Y)$. Thus, $Z \neq \emptyset$. Let $\hat{Z}=\left\{k_{i j} \in Z\right.$ : $\left.(k-1)_{i j} \notin Z\right\}$. Thus, $\hat{Z}$ collects the "additional" goods provided in $Z$ compared to $Y$.

Because $Z \neq \emptyset$ and $Z \cap Y=\emptyset$, we have $\hat{Z} \neq \emptyset$. Thus, there exist $i, j \in N(Z)$ such that $i \neq j$ and $k_{i j} \in \hat{Z}$. If $\hat{Z}_{\rightarrow j}=\emptyset$, then $Z_{\rightarrow j}=\emptyset$ and $Y_{i} \in C_{i}(Y \cup Z$ ) (as $j$ does not get additional goods in $Z$ ), a contradiction. Thus, $\hat{Z}_{\rightarrow j} \neq \emptyset$ and $h_{j l} \in \hat{Z}$ for some $l \in N(Z)$, and so on. But then we must a find a cycle $i_{1}, \ldots, i_{m}$ such that $k_{i_{1} i_{2}}^{1} \in \hat{Z}, \ldots, k_{i_{m} i_{1}}^{m} \in \hat{Z}$. But now $Y \cup\left\{k_{i_{1} i_{2}}^{1}, \ldots, k_{i_{m} i_{1}}^{m}\right\}$ is a feasible and individually rational allocation, a contradiction to the maximality of $Y$.

Proof of Lemma 2. We only show that condition (1) holds and remark that condition (2) can be shown similarly. Let $Y, Z \subseteq \mathcal{Y}_{i}$ be such that $Y_{i \rightarrow}=Z_{i \rightarrow}$ and $Y_{\rightarrow i} \subseteq Z_{\rightarrow i}$. Let $Y^{*} \in C_{i}(Y)$. Then (iii) is trivially satisfied as $\left|Y_{\rightarrow i}^{*}\right|-\left|Y_{i \rightarrow}^{*}\right|=0=\left|Z_{\rightarrow i}^{*}\right|-\left|Z_{i \rightarrow}^{*}\right|$ for all $Z^{*} \in C_{i}(Z)$. 
If for some $Z^{*} \in C_{i}(Z), U_{i}\left(Y^{*}\right)=U_{i}\left(Z^{*}\right)$, then $Y^{*} \in C_{i}(Z)$ and (i) and (ii) hold trivially. Otherwise, for some $Z^{*} \in C_{i}(Z), U_{i}\left(Y^{*}\right)<U_{i}\left(Z^{*}\right)$. But then choose $U_{i}\left(Z^{*}\right)-U_{i}\left(Y^{*}\right)$ contracts from $Z^{*} \backslash Y^{*}$, say the set $\hat{Z}$, such that $\left|\hat{Z}_{\rightarrow i}\right|=\left|\hat{Z}_{i \rightarrow \mid}\right|, k_{i j} \in \hat{Z}$ with $k>1$ implies $(k-1)_{i j} \in \hat{Z} \cup Y^{*}$, and $k_{j i} \in \hat{Z}$ with $k>1$ implies $(k-1)_{j i} \in \hat{Z} \cup Y^{*}$. Then we obtain $Y^{*} \cup \hat{Z} \in C_{i}(Z)$ (because $U_{i}\left(Y^{*} \cup \hat{Z}\right)=U_{i}\left(Z^{*}\right)$ ), and (i), (ii) and (iii) holds for $Y^{*}$ and $Y^{*} \cup \hat{Z}$.

\section{Appendix B: Proof of Theorem 1}

This Appendix first introduces a graph theoretical tool, referred to as the circulation-based model (Appendix B.1). It will then be demonstrated that the graph formulation in the circulation-based model, without loss of generality, can replace the min-cost flow problem when analyzing the priority mechanism (Appendix B.2). These insights enable us to prove that the flow does not increase when a capacity is reduced on an arc in the graph of the circulation-based model. This is the key step in the proof of Theorem 1 (Appendix B.3).

\section{Appendix B.1: The Circulation-Based Model}

Let $\mathbb{Z}$ denote the set containing all integers. For any profile $R \in \tilde{\mathcal{R}}$, construct a weighted directed graph $D_{R}=(V, A)$ with capacities $c: A \mapsto \mathbb{N}_{0}$ and weights $w: A \mapsto \mathbb{Z}$ on its arcs. For ease of notation, we write $D$ instead of $D_{R}$ whenever the profile $R$ is unambiguous. Each agent $i \in N$ is represented by two vertices, denoted by $i^{\text {in }}$ and $i^{\text {out }}$. These $2 n$ vertices build the vertex set $V$ of the graph $D$. We draw a directed arc between each pair of type $\left(i^{\text {in }}, i^{\text {out }}\right)$, pointing to $i^{\text {out }}$ and refer to this arc as the inner arc of agent $i \in N$. The inner arc has capacity $c\left(i^{i n}, i^{\text {out }}\right)=t_{i}$. If agent $i$ finds agent $j$ acceptable, then $\left(j^{\text {out }}, i^{i n}\right)$ belongs to the (directed) arc set $A$ of the graph $D$. Any such arc is called regular and has capacity $c\left(j^{\text {out }}, i^{i n}\right)=\bar{t}_{i j}$, i.e., the upper bound on the number of goods that agent $i$ would like to receive from agent $j$. Note also that the vertices of type $i^{i n}$ have incoming regular arcs and a single outgoing inner arc, while vertices of type $i^{\text {out }}$ have outgoing regular arcs and a single incoming inner arc. We define in Appendix B.2 the weights $w: A \mapsto \mathbb{Z}$ using a priority-order. An instance of the model is illustrated in Figure 2 (the figure contains some concepts which are explained later in the Appendix).

Definition 5. A circulation is a function $C: A \mapsto \mathbb{N}_{0}$ where:

(i) $C(u, v) \leq c(u, v)$ for every $(u, v) \in A$, 
(ii) $\sum_{(u, v) \in A} C(u, v)=\sum_{(v, w) \in A} C(v, w)$ for every vertex $v \in V$.

Condition (i) is a capacity constraint which ensures that agents do not supply goods beyond their endowment $t_{i}=c\left(i^{i n}, i^{\text {out }}\right)$, and that the upper bound $\bar{t}_{i j}$ is not exceeded. Condition (ii) is the classical flow conservation rule, stating that the total flow of the incoming arcs of a vertex equals the total flow of the outgoing arcs, i.e., that an agent supplies and receives the same number of goods. The latter condition can also be formulated as:

$$
C\left(i^{\text {in }}, i^{\text {out }}\right)=\sum_{\left(j^{\text {out }}, i^{\text {in }}\right) \in A} C\left(j^{\text {out }}, i^{\text {in }}\right)=\sum_{\left(i^{\text {out }}, k^{\text {in }}\right) \in A} C\left(i^{\text {out }}, k^{\text {in }}\right) \text { for every agent } i \in N \text {. }
$$

Note that a circulation is by definition a network flow. We call $C\left(i^{\text {in }}, i^{\text {out }}\right)$ the flow value at agent $i$. Circulations in a graph $D$ are in one-to-one correspondence with allocations in the exchange problem, e.g., for an allocation $x$ the corresponding flow value of the inner arc at agent $i$ is $C\left(i^{i n}, i^{\text {out }}\right)=t_{i}-x_{i i}$ and the flow value of any regular arc at agent $i$ is $C\left(j^{\text {out }}, i^{i n}\right)=x_{i j}$ for all $j \in N$. The allocation value for agent $i$ is defined as $t_{i}-x_{i i}$. Another way of expressing this is that the allocation value $t_{i}-x_{i i}$ of agent $i$ in the exchange problem equals the flow value $C\left(i^{i n}, i^{\text {out }}\right)$ at agent $i$ in the circulation model.

\section{Appendix B.2: Replacement Result}

This section demonstrates that by placing appropriate weights on the $\operatorname{arcs}$ in the graph $D$, the maximum weight circulations correspond to the outcome of the min-cost flow problem used in Section 5 to identify the outcome of the priority mechanism (Proposition 2). This result implies that the circulation-based model can be adopted in the proof of Theorem 1. We remark that both maximum weight circulations and min-cost flows can be computed efficiently, or more precisely, in $O\left(|E|^{2} \log |V|\right)$ time in a graph with $|V|$ vertices and $|E|$ arcs , which is strongly polynomial time (Orlin, 1993).

Let $\pi$ be a priority-ordering. Let $t_{\max }$ be the largest endowment of any agent in $N$, and define the weight $w(u, v)$ on each $\operatorname{arc}(u, v)$ in the directed graph $D=(V, A)$ by:

$$
w(u, v)= \begin{cases}t_{\max }^{2(n+1-\pi(i))} & \text { if }(u, v)=\left(i^{\text {in }}, i^{\text {out }}\right) \\ 0 & \text { otherwise. }\end{cases}
$$

We now illustrate our transformation from the priority mechanism to the circulation-based model by 
means of a simple example.

Example 4. Agents are denoted by $i, j, k$ and $l$. The upper bounds on the acceptable goods are as follows: $\bar{t}_{k i}=1, \bar{t}_{j i}=2, \bar{t}_{k j}=2, \bar{t}_{l j}=1, \bar{t}_{i k}=3, \bar{t}_{i l}=1, \bar{t}_{j l}=3$. All other upper bounds are set to 0 . Each agent has an endowment of 3, and their priority-order is alphabetic.

In the circulation-based model, there are two vertex copies to each agent, connected by an inner arc. The number of goods that each agent is willing to accept from another agent translates into an upper capacity on the regular arc connecting the out-vertex of the provider and the in-vertex of the receiver. In Figure 2, inner arcs are marked by horizontal lines, while regular arcs are bent and colored. Arc weights and capacities are written above and below each arc, respectively. Due to the alphabetic priority-order and $t_{\max }$ being 3 units, the arc weights of agents $i, j, k$ and $l$ on the inner arcs are given by $3^{8}, 3^{6}, 3^{4}$ and $3^{2}$, respectively. All arc weights on regular arcs are set to zero.

The max weight circulation in the network can be computed efficiently, and it has weight $3 \cdot 3^{8}+$ $3 \cdot 3^{6}+3 \cdot 3^{4}+1 \cdot 3^{2}$. It saturates all edges except the dotted $\left(l^{\text {out }}, i^{\text {in }}\right)$ which is left empty, and the dashed $\left(l^{\text {out }}, j^{\text {out }}\right)$ and $\left(l^{\text {in }}, i^{\text {out }}\right)$, both of which carry one unit of flow. More precisely, agent $i$ sends 2 units of his good to agent $j$ and 1 unit of his good to agent $k$, agent $j$ sends 2 units of his good to agent $k$ and 1 unit of his good to agent $l$, agent $k$ sends 3 units of his good to agent $i$, and agent $l$ sends 1 unit of his good to agent $j$.

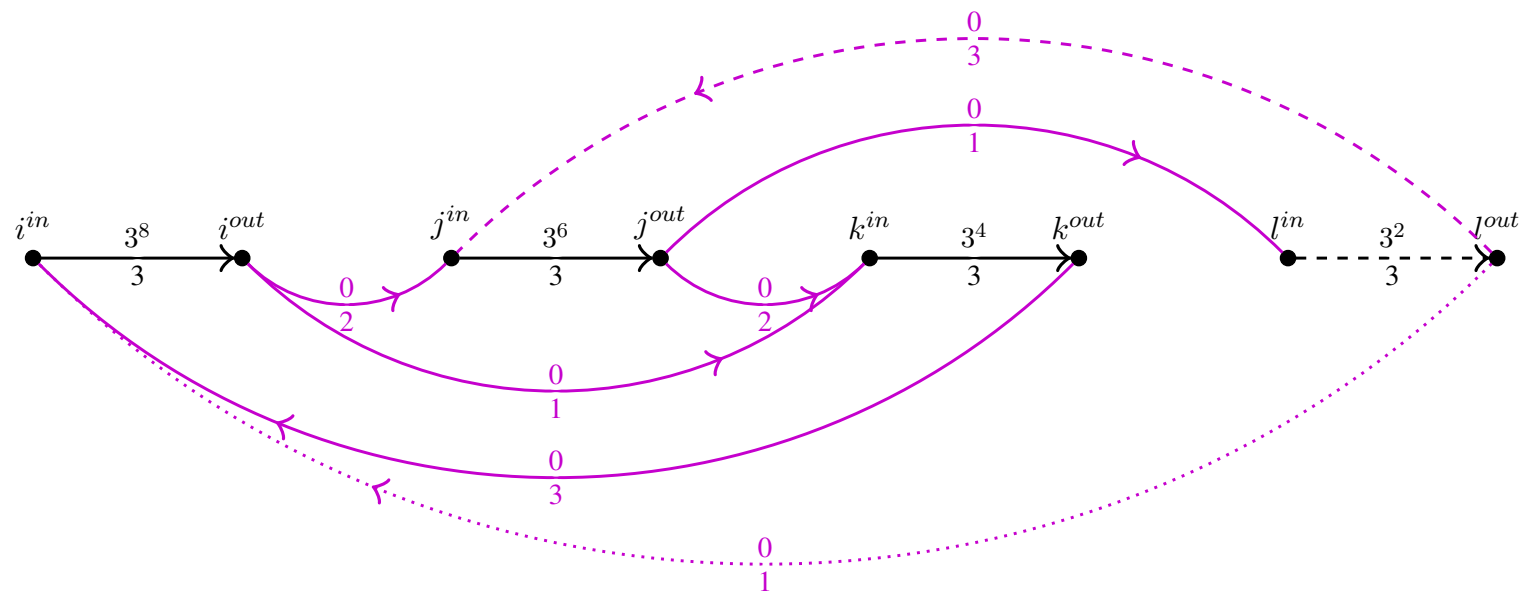

Figure 2: The circulation-based model for the instance in Example 4.

Let $w(C)$ denote the weighted sum of flow values of the agents in $N$ at circulation $C$, i.e., $w(C)=$ $\sum_{i \in N} C\left(i^{\text {in }}, i^{\text {out }}\right) \cdot w\left(i^{\text {in }}, i^{\text {out }}\right)$. 
Proposition 2. For any given profile $R \in \tilde{\mathcal{R}}$, let $C$ be a maximum weight circulation where the weights are defined by condition (9). Let $C^{\prime}$ be the circulation corresponding to an allocation $x^{\prime}$ selected for $R$ by a priority mechanism $\varphi$ based on $\pi$. Then $C^{\prime}\left(i^{\text {in }}, i^{\text {out }}\right)=C\left(i^{\text {in }}, i^{\text {out }}\right)$ for each $i \in N$.

Proof. As in the statement of the proposition, let $C$ be a maximum weight circulation and let $C^{\prime}$ be the circulation corresponding to an allocation $x^{\prime}$ selected by a priority mechanism. Suppose, to obtain a contradiction, that $C^{\prime}\left(j^{\text {in }}, j^{\text {out }}\right) \neq C\left(j^{\text {in }}, j^{\text {out }}\right)$ for some $j \in N$. Let agent $i$ be the agent with the highest priority in $\pi$ where this holds. Suppose also, without loss of generality, that $\pi(k)=k$ for all $k \in N$. To reach the desired contradiction, we consider two cases.

Case (i): $C^{\prime}\left(i^{\text {in }}, i^{\text {out }}\right)<C\left(i^{\text {in }}, i^{\text {out }}\right)$. In this case, the maximum weight circulation $C$ assigns a higher allocation value to agent $i$ than the priority mechanism. We show by induction that this contradicts the rules of the priority mechanism. Suppose first that agent $i$ is the highest-ranked agent according to the priority-order $\pi$ and recall that the priority mechanism, by construction, restricts the set of maximal allocations to those that maximize the allocation value of $i$ (see condition (3) in Section 4). Thus, there is no allocation that assigns agent $i$ a higher allocation value than the allocations in this chosen set, and, consequently, no circulation that assigns agent $i$ a higher value. Hence, agent $i$ cannot be the agent with the highest priority. Suppose now that agent $i$ is the secondhighest-ranked agent according to the priority-order $\pi$. Again, by condition (3) this agent restricts the set of allocations further. And so, the maximum weight circulation $C$ is still in the chosen set when agent $i$ restricts the set of allocations further, and it can, consequently, not have a higher allocation value for agent $i$ than $C^{\prime}$. This argument can be repeated inductively to reach the conclusion that it cannot be the case that $C^{\prime}\left(i^{\text {in }}, i^{\text {out }}\right)<C\left(i^{\text {in }}, i^{\text {out }}\right)$.

Case (ii): $C^{\prime}\left(i^{\text {in }}, i^{\text {out }}\right)>C\left(i^{\text {in }}, i^{\text {out }}\right)$. Note first that both $C$ and $C^{\prime}$ are feasible circulations at profile $R$. Because agent $i$ is the agent with the highest priority in $\pi$ where $C^{\prime}\left(i^{\text {in }}, i^{\text {out }}\right) \neq C\left(i^{\text {in }}, i^{\text {out }}\right)$, by assumption, it follows that $C^{\prime}\left(k^{\text {in }}, k^{\text {out }}\right)=C\left(k^{\text {in }}, k^{\text {out }}\right)$ for all agents $k=1, \ldots, i-1$. It will be demonstrated that agents $i+1, \ldots, n$ cannot make up for the loss $C$ suffered on $\operatorname{arc}\left(i^{\text {in }}, i^{\text {out }}\right)$ and thus $C$ cannot be of maximum weight since $C^{\prime}$ is a feasible circulation at profile $R$. Recall first that the set $\mathbb{N}_{0}$ contains only positive integers, so the difference between $C^{\prime}\left(i^{\text {in }}, i^{\text {out }}\right)$ and $C\left(i^{\text {in }}, i^{\text {out }}\right)$ is at least 1 . By construction of the weights on the inner arcs, defined by condition (9), it then follows that:

$$
\left[C^{\prime}\left(i^{\text {in }}, i^{\text {out }}\right)-C\left(i^{\text {in }}, i^{\text {out }}\right)\right] \cdot t_{\max }^{2(n-i+1)} \geq t_{\max }^{2(n-i+1)} .
$$


Note next that, in the extreme case, all agents with lower priorities than agent $i$ have flow value zero in $C^{\prime}$ and a flow value of $t_{\max }$ in $C$. This means that the weighted sum of the flow values at agents $i+1, \ldots, n$ at circulation $C$ is at most:

$$
t_{\max } \cdot \sum_{j=i+1}^{n} t_{\max }^{2(n-j+1)} .
$$

Now, the value of the sum (11) is strictly lower than the right hand side of inequality (10). Consequently, even in the extreme case when all agents with lower priorities than agent $i$ have flow value zero in $C^{\prime}$ and a flow value of $t_{\max }$ in $C$, it holds that $w\left(C^{\prime}\right)>w(C)$. However, this contradicts that $C$ is a maximum weight circulation since $C^{\prime}$ is a feasible circulation at graph $D_{R}$.

\section{Appendix B.3: The Proof}

Let $\varphi$ be the priority mechanism based on $\pi$ where $\pi(i)=i$ for all $i \in N$. To obtain a contradiction, suppose that $\varphi$ can be manipulated by some agent $i \in N$ at a profile $R \in \tilde{\mathcal{R}}$. This means that there are two profiles $R \in \tilde{\mathcal{R}}$ and $R^{\prime}=\left(R_{i}^{\prime}, R_{-i}\right) \in \tilde{\mathcal{R}}$ such that for $x=\varphi(R)$ and $x^{\prime}=\varphi\left(R^{\prime}\right)$ we have $x_{i}^{\prime} P_{i} x_{i}$. Note that $R_{i}^{\prime} \neq R_{i}$. Let $C^{1}$ and $C^{2}$ be the maximum weight circulations for the graphs $D_{R}$ and $D_{R^{\prime}}$ induced by the profiles $R$ and $R^{\prime}=\left(R_{i}^{\prime}, R_{-i}\right)$, respectively.

The next lemma shows that we may suppose that the set of acceptable agents reported by agent $i$ at preference relation $R_{i}^{\prime}$ is a proper subset of the set of acceptable agents reported by agent $i$ at preference relation $R_{i}$.

Lemma 3. Without loss of generality, we may suppose $A_{i}\left(R_{i}^{\prime}\right) \subseteq A_{i}\left(R_{i}\right)$.

Proof. We first show $U_{i}\left(R_{i}\right) \subseteq U_{i}\left(R_{i}^{\prime}\right)$. To see this, suppose $j \in U_{i}\left(R_{i}\right)$ but $j \notin U_{i}\left(R_{i}^{\prime}\right)$, i.e., that agent $j$ is unacceptable under $R_{i}$ but acceptable under $R_{i}^{\prime}$. Since $x_{i}^{\prime} P_{i} x_{i}$, it must then hold that $x_{i j}^{\prime}=0$ by definition of the preferences in $\tilde{\mathcal{R}}_{i}$. Hence, any regular arc of type $\left(j^{\text {out }}, i^{\text {in }}\right)$ where $j \notin U_{i}\left(R_{i}^{\prime}\right)$ in the graph $D_{R^{\prime}}$ but $j \in U_{i}\left(R_{i}\right)$ in the graph $D_{R}$ will not be active in the solution $C^{1}$ at profile $R^{\prime}$. Hence, $U_{i}\left(R_{i}\right) \subseteq U_{i}\left(R_{i}^{\prime}\right) \cup\left\{j \in A_{i}\left(R_{i}^{\prime}\right): x_{i(n+j)}^{\prime}=0\right\}$.

But then we may choose $R_{i}^{\prime \prime}$ such that $A_{i}\left(R_{i}^{\prime \prime}\right)=A_{i}\left(R_{i}^{\prime}\right) \backslash\left\{j \in A_{i}\left(R_{i}^{\prime}\right): x_{i(n+j)}^{\prime}=0\right\}$ and $\bar{t}_{i k}^{\prime \prime}=\bar{t}_{i k}^{\prime}$ for all $k \in A_{i}\left(R_{i}^{\prime \prime}\right)$, and $C^{1}$ remains a solution for $R^{\prime \prime}=\left(R_{i}^{\prime \prime}, R_{-i}\right) \in \tilde{\mathcal{R}}$. But for $x^{\prime \prime}=\varphi\left(R^{\prime \prime}\right)$ this implies $x_{i}^{\prime \prime} I_{i} x_{i}^{\prime}$ and $x_{i}^{\prime \prime} P_{i} x_{i}$. Hence, $A_{i}\left(R_{i}^{\prime \prime}\right) \subseteq A_{i}\left(R_{i}\right)$ and $x_{i}^{\prime \prime} P_{i} x_{i}$.

Recall now that, for any profile in $R \in \tilde{\mathcal{R}}$, each agent $k \in N$ reports a set of acceptable agents $A_{k}\left(R_{k}\right)$ together with an upper bound $\bar{t}_{k j}$ on the number of goods that agent $k \in N$ at most would 
like to receive from each acceptable agent $j \in A_{k}\left(R_{k}\right)$. By Remark 1, the report $R_{k}$ is equivalent to the vector $\bar{t}_{k}$ where $\bar{t}_{k k}=t_{k}$ and $\bar{t}_{k j}=0$ for all $j \in U_{k}\left(R_{k}\right)$. This, together with the conclusion in Lemma 3, implies that there exists at least one agent $j$ that is acceptable for agent $i$ under $R_{i}$ where agent $i$ reports a strictly lower or higher bound $\bar{t}_{i j}^{\prime}$ at profile $R^{\prime}$ than under profile $R$ (i.e., $\bar{t}_{i j}^{\prime}<\bar{t}_{i j}$ or $\bar{t}_{i j}^{\prime}>\bar{t}_{i j}$ ). In general, a manipulation $R_{i}^{\prime}$ by agent $i$ can consist of both underreporting and overreporting $\bar{t}_{i j}$ 's for acceptable agents. There are two possible cases for manipulations: one with overreporting and the other with only underreporting bounds. We will first show that overreporting any bound cannot be beneficial, and then we prove that no combination of underreporting bounds can result in a better allocation either.

First, consider the case where there is overreporting. If there exists $j \in N \backslash\{i\}$ such that $x_{i j}^{\prime}>\bar{t}_{i j}$, then by definition of $\tilde{\mathcal{R}}_{i}, \omega_{i} P_{i} x_{i}^{\prime}$ and since $x$ is individually rational under $R$, we have $x_{i} P_{i} x_{i}^{\prime}$, a contradiction. Otherwise $x_{i j}^{\prime} \leq \bar{t}_{i j}$ for all $j \in N \backslash\{i\}$ and we can just replace $\bar{t}_{i}^{\prime}$ with $\bar{t}_{i}^{\prime \prime}$ such that $\bar{t}_{i j}^{\prime \prime}=\min \left\{\bar{t}_{i j}, \bar{t}_{i j}^{\prime}\right\}$ for all $j \in N \backslash\{i\}$. Let $R_{i}^{\prime \prime}$ denote $i$ 's preference associated with $\bar{t}_{i}^{\prime \prime}$. Then $x^{\prime}$ is still a maximizer for the profile $\left(R_{i}^{\prime \prime}, R_{-i}\right)$ and therefore the manipulation only consists of underreporting upperbounds which are below $\bar{t}_{i}$.

Second, it remains to establish that agent $i$ cannot manipulate by underreporting the upper bounds for acceptable agents, i.e., that $\bar{t}_{i j}^{\prime} \leq \bar{t}_{i j}$ for all $j \in N \backslash\{i\}$. Below we are going to show that agent $i$ cannot gain by underreporting one upper bound for an acceptable agent. This is enough to establish that agent $i$ never can gain by reporting a lower bound for several agents at the same time. Because any such misreport can be decomposed into a sequence of manipulations in which at each step only one upper bound $\bar{t}_{i j}$ is changed at the time and agent $i$ is never made better off at any step. Formally, let $k \in A_{i}\left(R_{i}\right)$ for which $\bar{t}_{i k}^{\prime}<\bar{t}_{i k}$ and consider the misreport $\bar{t}_{i}^{(1)}$ where $\bar{t}_{i j}^{(1)}=\bar{t}_{i j}$ for all $j \neq k$ and $\bar{t}_{i k}^{(1)}=\bar{t}_{i k}^{\prime}$. Let $x^{(1)}$ be the allocation chosen by the priority mechanism when $i$ reports $t^{(1)}$. Below we show that agent $i$ cannot gain by reporting $\bar{t}_{i}^{(1)}$ instead of $\bar{t}_{i}$. In particular, $\sum_{j \in A_{i}\left(R_{i}\right)} x_{i j} \geq \sum_{j \in A_{i}\left(R_{i}\right)} x_{i j}^{(1)}$. Thus, $x_{i} R_{i} x_{i}^{(1)}$. If there is another agent $\ell \neq k$ such that $\bar{t}_{i \ell}^{(1)} \neq \bar{t}_{i \ell}^{\prime}$ then consider $\bar{t}^{(2)}$ where $\bar{t}_{i j}^{(2)}=\bar{t}_{i j}^{(1)}$ for all $j \neq \ell$ and $\bar{t}_{i \ell}^{(2)}=\bar{t}_{i \ell}^{\prime}$. Suppose again that agent $i$ cannot gain by reporting $\bar{t}_{i}^{(2)}$ instead of $\bar{t}_{i}^{(1)}$. This means again that $\sum_{j \in A_{i}\left(R_{i}\right)} x_{i j}^{(1)} \geq \sum_{j \in A_{i}\left(R_{i}\right)} x_{i j}^{(2)}$. Thus, by transitivity $x_{i} R_{i} x^{(2)}$. This argument can be repeated inductively until the point that $\bar{t}_{i}^{(p)}=\bar{t}_{i}^{\prime}$, and if in each step agent $i$ never gains by reporting $\bar{t}_{i}^{(j)}$ instead of $\bar{t}^{(j-1)}$ we have shown that agent $i$ cannot gain by reporting $\bar{t}_{i}^{\prime}$ instead of $\bar{t}_{i}$. Hence, to complete the proof of Theorem 1 , it is enough to show that agent $i$ cannot gain by misreporting $\bar{t}_{i j}^{\prime}$ for one agent $j \in A_{i}\left(R_{i}\right)$. 
Thus, to prove non-manipulability of the priority mechanism, it only remains to rule out that agent $i$ cannot gain by reporting a strictly lower bound $\bar{t}_{i j}$. Translating this into the terminology of the circulation-based model, this can equivalently be expressed as the flow value $C\left(i^{\text {in }}, i^{\text {out }}\right)$ at agent $i$ in a maximum weight circulation cannot be increased by reducing the capacity on a regular arc $\left(j^{\text {out }}, i^{\text {in }}\right)$. Given this insight, a large part of the remaining proof will focus on a regular $\operatorname{arc}\left(j^{\text {out }}, i^{\text {in }}\right)$.

Recall now that $C^{1}$ denotes the maximum weight circulations for the true preferences $R$ induced by the graph $D_{R}$, and that $C^{2}$ denotes the maximum weight solution for the misrepresented preferences $R^{\prime}$ induced by the graph $D_{R^{\prime}}$. Furthermore, by the assumption that agent $i$ can manipulate the priority mechanism, it follows that $C^{2}$ has a larger flow value at agent $i$ than $C^{1}$ does, i.e., $C^{2}\left(i^{\text {in }}, i^{\text {out }}\right)>C^{1}\left(i^{\text {in }}, i^{\text {out }}\right)$. By construction of the weights in condition (9), the circulation value of $C^{2}$ cannot be the same as the circulation value of $C^{1}$ if the flow value differs for at least one agent. Thus, the circulation value of $C^{2}$ must be strictly smaller than the circulation value of $C^{1}$, i.e., $w\left(C^{2}\right)<w\left(C^{1}\right)$. Note also that the circulation $C^{2}$ is a feasible circulation in $D_{R}$ since the flows remain below the capacity on each arc, and it preserves flow conservation. However, the circulation $C^{2}$ is not optimal in the graph $D_{R}$ since the circulation value of $C^{2}$ is strictly smaller than the circulation value of $C^{1}$, and the circulation $C^{1}$ is optimal in $D_{R}$.

Consider next the function defined by the circulation $C^{1}-C^{2}$ where $C^{1}(u, v)-C^{2}(u, v) \in \mathbb{Z}$ for each $\operatorname{arc}(u, v)$ in the graph $D_{R}$. This function assigns a negative value to the $\operatorname{arc}(u, v)$ if the flow through the arc is larger in circulation $C^{2}$ than in circulation $C^{1}$. For convenience, one can think of these "negative" arcs as arcs turned backwards, with the usual positive flow value on them. Since both $C^{1}$ and $C^{2}$ are circulations in the graph $D_{R}$, their difference also obeys flow conservation, and, as such, it can be decomposed into cycles.

A cycle decomposition is a collection of directed cycles in the graph so that the flow value on all edges of a specific cycle in the decomposition is the same, and the sum of flow values in all cycles containing an $\operatorname{arc}(u, v)$ equals the flow value on $(u, v)$. The capacity or the weight on the edges plays no role in the decomposition. It is known that any feasible circulation has a cycle decomposition (Ford and Fulkerson, 1956). In the next paragraph, we will construct such a cycle decomposition of the circulation $C^{1}-C^{2}$. For simplicity, we will decompose our circulation into cycles of flow value 1 .

Note first that a cycle decomposition of $C^{1}-C^{2}$ need not be unique for the profiles $R$ and $R^{\prime}$. To obtain one such decomposition, we use a simple inductive algorithm that produces a cycle decomposition of $C^{1}-C^{2}$ in a finite number of iterations. This algorithm uses the flow value of 
$C^{1}-C^{2}$ on each arc $(u, v)$ in the graph $D_{R}$ but will not use any information about the arc capacities or weights (arc weights are only considered below). First, identify any directed cycle, say $\mathcal{C}$, based on the circulation $C^{1}-C^{2}$ and take its forward or backward arc with a lowest absolute flow value on it. Suppose that the lowest absolute flow value at some agent in the cycle $\mathcal{C}$ is $q$, then $q$ feasible cycles of type $\mathcal{C}$ can be identified. These cycles represent the first $q$ cycles in the decomposition of $C^{1}-C^{2}$. Then, reduce the flow value on each arc included in the cycle $\mathcal{C}$ by $q$. This will give an "updated" circulation, based on the "original" circulation $C^{1}-C^{2}$. Notice that the updated circulation is indeed a circulation, preserving flow conservation at each vertex, but, compared to $C^{1}-C^{2}$, it is guaranteed to have at least one more arc with zero flow value. We proceed in this manner until the whole circulation $C^{1}-C^{2}$ is decomposed into cycles. Note also that, since $\mathbb{N}_{0}$ is restricted to a set of positive bounded integers, this procedure ends in a finite number of iterations. Moreover, the absolute flow value on an arc monotonically (but not strictly monotonically) decreases in each inductive step, until it reaches 0 .

Note that the cycles in the decomposition are not necessarily arc-disjoint from each other (i.e., several distinct cycles in the decomposition can pass through the same arc), but, due to the inductive argument above, each arc in the cycle decomposition is either a forward arc or a backward arc, depending on the sign of $C^{1}(u, v)-C^{2}(u, v)$. More precisely, forward arcs are positive, while backward arcs are negative. Thus, it cannot be the case that one cycle in the decomposition uses an arc with positive value, while another cycle uses the same arc with negative value.

Consider now the cycle decomposition of the circulation $C^{1}-C^{2}$ as constructed above. We now turn towards arc weights: the total weight of a cycle in the decomposition is defined as the sum of weights on each arc in the cycle. Based on the sign of the total weight of a cycle, we distinguish positive, zero and negative weight cycles in our decomposition. A positive weight cycle is called an augmenting cycle. Note that all augmenting cycles pass through $\left(j^{\text {out }}, i^{\text {in }}\right)$, because any augmenting cycle which does not pass through $\left(j^{\text {out }}, i^{\text {in }}\right)$ would increase the circulation value of $C^{2}$ in $D_{R^{\prime}}$, which is impossible since $C^{2}$ is optimal in the graph $D_{R^{\prime}}$.

Lemma 4. Suppose that $C^{1}-C^{2}$ is decomposed into cycles using the inductive decomposition algorithm from above. Then:

(i) there exists an augmenting cycle,

(ii) a cycle of weight zero consists exclusively of arcs of weight zero, 
(iii) there are no negative weight cycles.

Proof. The proof of Part (i) follows directly since $w\left(C^{1}\right)>w\left(C^{2}\right)$ and $w\left(C^{1}\right)$ equals $w\left(C^{2}\right)$ plus the weight of each cycle in the cycle decomposition of $C^{1}-C^{2}$. Part (ii) follows by construction of the weights, i.e., a cycle of weight zero consists exclusively of arcs of weight zero (obviously, no combination of the weights on inner arcs with coefficients in the open interval between 0 and $t_{\max }$ can add up to zero).

Part (iii) is proved by contradiction. Suppose that there is a cycle $\mathcal{C}$ of negative total weight in the cycle decomposition of $C^{1}-C^{2}$. Let the reverse of $\mathcal{C}$ be denoted by $\overleftarrow{\mathcal{C}}$. The reverse $\overleftarrow{\mathcal{C}}$ has positive total weight and preserves the sign of $C^{2}-C^{1}$ on each of its arcs by construction of the inductive decomposition algorithm. Moreover, we will show that, $\overleftarrow{\mathcal{C}}$ can be added to $C^{1}$ without violating flow conservation or any capacity constraint in $D_{R}$. Thus, $C^{1}+\overleftarrow{\mathcal{C}}$ is a circulation of larger weight than $C^{1}$. Let now $(u, v)$ be an arbitrary arc in the reverse cycle $\overleftarrow{\mathcal{C}}$. It will be demonstrated that:

$$
0 \leq C^{1}(u, v)+\overleftarrow{\mathcal{C}}(u, v) \leq c(u, v)
$$

Condition (12) implies that $C^{1}$ cannot be a maximum weight circulation in the graph $D_{R}$ which contradicts our assumption. We need to consider two cases. Suppose first that $\overleftarrow{\mathcal{C}}(u, v) \geq 0$. Then:

$$
C^{1}(u, v)+\overleftarrow{\mathcal{C}}(u, v) \leq C^{1}(u, v)+\left[C^{2}(u, v)-C^{1}(u, v)\right]=C^{2}(u, v) \leq c(u, v)
$$

Note also that because $C^{1}(u, v)$ and $\overleftarrow{\mathcal{C}}(u, v)$ are non-negative at the arc $(u, v)$, it follows directly that $C^{1}(u, v)+\overleftarrow{\mathcal{C}}(u, v) \geq 0$. Hence, condition (12) holds when $\overleftarrow{\mathcal{C}}(u, v) \geq 0$. Suppose next that $\overleftarrow{\mathcal{C}}(u, v)<0$. In this case:

$$
C^{1}(u, v)+\overleftarrow{\mathcal{C}}(u, v)<C^{1}(u, v) \leq c(u, v)
$$

Furthermore:

$$
C^{1}(u, v)+\overleftarrow{\mathcal{C}}(u, v) \geq C^{1}(u, v)+\left[C^{2}(u, v)-C^{1}(u, v)\right]=C^{2}(u, v) \geq 0
$$

Hence, condition (12) also holds when $\overleftarrow{\mathcal{C}}(u, v)<0$

Lemma 4 thus demonstrated that all cycles in the cycle decomposition of $C^{1}-C^{2}$, which pass through 
an inner arc, are augmenting cycles. However, we do not know whether these cycles use the arc $\left(j^{\text {out }}, i^{\text {in }}\right)$ as a forward arc or as a backward arc. The following lemma sheds light on this.

Lemma 5. Suppose that $C^{1}-C^{2}$ is decomposed into cycles using the inductive decomposition algorithm from above, and let $\left(j^{\text {out }}, i^{\text {in }}\right)$ be an arbitrary arc in some cycle in the cycle decomposition of $C^{1}-C^{2}$. Then $\left(j^{\text {out }}, i^{\text {in }}\right)$ is a forward arc.

Proof. Note first that $C^{2}\left(j^{\text {out }}, i^{\text {in }}\right)$ is bounded from above by the decreased capacity of $\left(j^{\text {out }}, i^{\text {in }}\right)$ in $D_{R^{\prime}}$. If $C^{1}\left(j^{\text {out }}, i^{\text {in }}\right) \leq C^{2}\left(j^{\text {out }}, i^{\text {in }}\right)$, then $C^{1}$ is feasible in the graph $D_{R^{\prime}}$ and has a larger weight than $C^{2}$, which contradicts the optimality of $C^{2}$ in the graph $D_{R^{\prime}}$. Thus, $C^{1}\left(j^{\text {out }}, i^{\text {in }}\right)-C^{2}\left(j^{\text {out }}, i^{\text {in }}\right)>0$, which implies that $\left(j^{\text {out }}, i^{\text {in }}\right)$ is a forward arc in all cycles in the decomposition of $C^{1}-C^{2}$.

Finally, consider the flow value $C^{1}\left(i^{\text {in }}, i^{\text {out }}\right)-C^{2}\left(i^{\text {in }}, i^{\text {out }}\right)$. To prove Theorem 1 , we only need to establish that $C^{1}\left(i^{\text {in }}, i^{\text {out }}\right)-C^{2}\left(i^{\text {in }}, i^{\text {out }}\right) \geq 0$ because this contradicts the assumption that $x_{i}^{\prime} P_{i} x_{i}$. For this condition to be false, the $\operatorname{arc}\left(i^{\text {in }}, i^{\text {out }}\right)$ must be a backward arc in at least one cycle in the cycle decomposition of $C^{1}-C^{2}$. However, as concluded above, being a backward arc in one cycle also implies being a backward arc in all cycles. From Lemma 4 we know that all cycles that pass through $\left(i^{\text {in }}, i^{\text {out }}\right)$ are augmenting cycles. Lemma 5 then states that the augmenting cycles use $\left(j^{\text {out }}, i^{\text {in }}\right)$ as a forward arc, and they must, consequently, leave $i^{i n}$ either as a forward arc, along the only outgoing $\operatorname{arc}\left(i^{i n}, i^{\text {out }}\right)$, or as a backward arc, along any of the regular arcs running to $i^{i n}$. Neither of these two cases allows $\left(i^{\text {in }}, i^{\text {out }}\right)$ to be a backward arc. This concludes the proof and shows that agent $i$ cannot manipulate the priority mechanism $\varphi$ at any profile $R \in \tilde{\mathcal{R}}$.

\section{References}

Abdulkadiroğlu, A. and T. Sönmez (1999). House allocation with existing tenants. Journal of Economic Theory 88, 233-260.

Abdulkadiroğlu, A. and T. Sönmez (2003). School choice: A mechanism design approach. American Economic Review 93, 729-747.

Alcalde, J. and S. Barberà (1994). Top dominance and the possibility of strategy-proof stable solutions to the marriage problem. Economic Theory 4, 417-435.

Alcalde-Unzu, J. and E. Molis (2011). Exchange of indivisible goods and indifferences: The top trading absorbing sets mechanisms. Games and Economic Behavior 73, 1-16. 
Athanassoglou, S. and J. Sethuraman (2011). House allocation with fractional endowments. International Journal of Game Theory 40, 481-513.

Aziz, H. (2016). Generalizing top trading cycles for housing markets with fractional endowments. arXiv:1509.03915.

Aziz, H. (2018). Mechanisms for house allocation with existing tenants under dichotomous preferences. The Journal of Mechanism and Institution Design 3(1), 97-110.

Barberà, S. and M. Jackson (1995). Strategy-proof exchange. Econometrica 63, 51-87.

Biró, P. (2017). Applications of matching models under preferences. In U. Endriss (Ed.), Trends in Computational Social Choice, Chapter 18, pp. 345-373. AI Access.

Biró, P., D. F. Manlove, and R. Rizzi (2009). Maximum weight cycle packing in directed graphs, with application to kidney exchange programs. Discrete Mathematics, Algorithms and Applications 1(04), 499-517.

Biró, P., S. Pápai, and F. Klijn (2017a). Balanced exchange in a multi-object Shapley-Scarf market. Working Paper.

Biró, P., S. Pápai, and F. Klijn (2017b). Serial dictatorships in a multi-object Shapley-Scarf market. Working Paper.

Bogomolnaia, A. and H. Moulin (2001). A new solution to the random assignment problem. Journal of Economic Theory 100, 295-328.

Bogomolnaia, A. and H. Moulin (2004). Random matching under dichotomous preferences. Econometrica $72,257-279$.

Boyle, D. and S. Bird (2014). Give and take: How timebanking is transforming healthcare. Gloucester: Timebanking UK.

Boyle, D., S. Clark, and S. Burns (2006). Co-production by people outside paid employment. The New Economic Foundation.

Cahn, E. (2000). No more throw-away people: The co-production imperative. Washington DC: Essential Books. 
Cahn, E. (2011). Time banking: An idea whose time has come? YES Magazine.

Caldwell, C. (2000). Why do people join local exchange trading systems. International Journal of Community Currency Research.

Collom, E. (2007). The motivations, engagement, satisfaction, outcomes, and demographics of time bank participants: Survey findings from a U.S. system. International Journal of Community Currency Research 11, 36-83.

Croall, J. (1997). LETS Act Locally: Growth of Local Exchange Trading Systems. Calouste Gulbenkian Foundation.

Dash, B. and N. Sandhu (2018). Time banking: The missing link. Development 61(1-4), 164-171.

Delacrétaz, D. (2019). Stability in matching markets with sizes. Working Paper.

Demange, G. (1987). Nonmanipulable cores. Econometrica 55, 1057-1074.

Dur, U. M. and O. Kesten (2018). Flexible top trading cycles and chains mechanism: Maintaining diversity in Erasmus student exchange. Working Paper.

Dur, U. M. and M. U. Ünver (2019). Two-sided matching via balanced exchange. Journal of Political Economy 127, 1156-1177.

Echenique, F. and J. Oviedo (2006). A theory of stability in many-to-many matching markets. Theoretical Economics 1, 233-273.

Ergin, H., T. Sönmez, and M. U. Ünver (2017). Dual-donor organ exchange. Econometrica 85(5), $1645-1671$.

Ford, L. and D. Fulkerson (1956). Maximal flow through a network. Canadian Journal of Mathematics $8,399-404$.

Gale, D. and L. S. Shapley (1962). College admissions and the stability of marriage. American Mathematical Monthly 69, 9-15.

Green, J. and J.-J. Laffont (1979). Incentives in public decision-making. Amsterdam: North-Holland.

Hatfield, J. W. and S. D. Kominers (2016). Contract design and stability in many-to-many matching. Games and Economic Behavior 101, 78-97. 
Hurwicz, L. (1972). On informationally decentralized systems. In C. McGuire and R. Radner (Eds.), Decision and Organization, pp. 297-336. North-Holland.

Jaramillo, P. and V. Manjunath (2012). The difference indifference makes in strategy-proof allocation of objects. Journal of Economic Theory 147, 1913-1946.

Kesten, O. and U. Ünver (2015). A theory of school-choice lotteries. Theoretical Economics 10, $543-595$.

Kominers, S. D., J. W. Hatfield, A. Nichifor, M. Ostrovsky, and A. Westkamp (2020). Chain stability in trading networks. Theoretical Economics. To appear.

Konishi, H., T. Quint, and J. Wako (2001). On the Shapley-Scarf Economy: The case of multiple types of indivisible goods. Journal of Mathematical Economics 35, 1-15.

Konishi, H. and M. U. Ünver (2006). Credible group-stability in many-to-many matching problems. Journal of Economic Theory 129, 57-80.

Manjunath, V. and A. Westkamp (2019). Strategy-proof exchange under under trichotomous preferences. Working Paper.

Orlin, J. B. (1993). A faster strongly polynomial minimum cost flow algorithm. Operations Research 41(2), 338-350.

Ozanne, L. K. (2010). Learning to exchagne time: Benefits and obstacles to time banking. International Journal of Community Currency Research 14, 1-16.

Roth, A. E. (1982). The economics of matching: Stability and incentives. Mathematics of Operations Research 7, 617-628.

Roth, A. E., T. Sönmez, and U. Ünver (2004). Kidney exchange. Quarterly Journal of Economics 119, $457-488$.

Scarf, H. and L. S. Shapley (1974). On cores and indivisibility. Journal of Mathematical Economics 1, $23-37$.

Schummer, J. (1999). Strategy-proofness versus efficiency for small domains of preferences over public goods. Economic Theory 13, 709-722. 
Seyfang, G. (2003). Growing cohesive communities one favour at a time: Social exclusion, active citizenship and time banks. International Journal of Urban and Regional Research 27, 699-706.

Seyfang, G. (2004). Time banks: Rewarding community self-help in the inner city? Community Development Journal 39, 62-71.

Seyfang, G. and K. Smith (2002). The time of our lives. The New Economic Foundation.

Sönmez, T. (1999). Strategy-proofness and essentially singled-valued cores. Econometrica 67, 677689.

Svensson, L.-G. (1994). Queue allocation of indivisible goods. Social Choice and Welfare 11, 323330.

Williams, C. C. (2004). Informal volunteering: Some lessons from the United Kingdom. Journal of Policy and Analysis and Management 23, 613-616. 